\title{
NKCC1 Activation Is Required for Myelinated Sensory Neurons Regeneration through JNK-Dependent Pathway
}

\author{
Laura Mòdol,^ Daniel Santos, ${ }^{\star}$ (DStefano Cobianchi, Francisco González-Pérez, Víctor López-Alvarez, \\ and Xavier Navarro \\ Institute of Neurosciences and Department of Cell Biology, Physiology and Immunology, Universitat Autònoma de Barcelona, and Centro de Investigación \\ Biomédica en Red sobre Enfermedades Neurodegenerativas, E-08193 Bellaterra, Spain
}

\begin{abstract}
After peripheral nerve injury, axons are able to regenerate, although specific sensory reinnervation and functional recovery are usually worse for large myelinated than for small sensory axons. The mechanisms that mediate the regeneration of different sensory neuron subpopulations are poorly known. The $\mathrm{Na}^{+}-\mathrm{K}^{+}-\mathrm{Cl}^{-}$cotransporter 1 (NKCC1) is particularly relevant in setting the intracellular chloride concentration. After axotomy, increased NKCC1 phosphorylation has been reported to be important for neurite outgrowth of sensory neurons; however, the mechanisms underlying its effects are still unknown. In the present study we used in vitro and in vivo models to assess the differential effects of blocking NKCC1 activity on the regeneration of different types of dorsal root ganglia (DRGs) neurons after sciatic nerve injury in the rat. We observed that blocking NKCC1 activity by bumetanide administration induces a selective effect on neurite outgrowth and regeneration of myelinated fibers without affecting unmyelinated DRG neurons. To further study the mechanism underlying NKCC1 effects, we also assessed the changes in mitogen-activated protein kinase (MAPK) signaling under NKCC1 modulation. The inhibition of NKCC1 activity in vitro and in vivo modified pJNK1/2/3 expression in DRG neurons. Together, our study identifies a mechanism selectively contributing to myelinated axon regeneration, and point out the role of $\mathrm{Cl}^{-}$modulation in DRG neuron regeneration and in the activation of MAPKs, particularly those belonging to the JNK family.
\end{abstract}

Key words: dorsal root ganglia; JNK signaling; MAPKs; myelinated fibers; NKCC1; regeneration

\section{Introduction}

Axonal breach upon injury is accompanied by changes in intracellular ion concentration in both the axon and soma of dorsal root ganglia (DRGs) primary afferent neurons (Bradke et al., 2012). These changes affect the function of ion channels that set and modulate neuronal responses, with alterations in the resting membrane potential, action potential initiation, or depolarization (Waxman and Zamponi, 2014). In this context, signaling in injured neurons is essential for the cell body transcription that modulates axonal regeneration and promotes the soma-axon communication by triggering a subset of events that occur after the lesion (Abe and Cavalli, 2008; Rishal and Fainzilber, 2010, 2014).

Plasma membrane cation- $\mathrm{Cl}^{-}$cotransporters are intrinsic membrane proteins that regulate the intracellular chloride con-

Received Oct. 2, 2014; revised April 4, 2015; accepted April 7, 2015.

Author contributions: L.M., S.C., and X.N. designed research; L.M., D.S., S.C., F.G.-P., V.L.-A., and X.N. performed research; L.M., D.S., S.C., and X.N. analyzed data; L.M., D.S., S.C., F.G.-P., and X.N. wrote the paper.

This work was supported by Red de Terapia Celular (TERCEL) and Centro de Investigación Biomédica en Red sobre Enfermedades Neurodegenerativas (CIBERNED) funds from the Fondo de Investigación Sanitaria of Spain, and Grant EPIONE (Grant 602547) from the European Community. The hybridoma antibody developed by Christian Lytle and Biff Forbush was obtained from the Developmental Studies Hybridoma Bank developed under the auspices of the NICHD and maintained by The University of lowa, Department of Biology (lowa City, IA). We thank Jessica Jaramillo, Marta Morell, and Mónica Espejo for technical help.

*L.M. and D.S. contributed equally to this work.

The authors declare no competing financial interests.

Correspondence should be addressed to Dr. Xavier Navarro, Unitat de Fisiologia Mèdica, Facultat de Medicina, Universitat Autònoma de Barcelona, E-08193 Bellaterra, Spain. E-mail: xavier.navarro@uab.cat.

DOI:10.1523/JNEUROSCI.4079-14.2015

Copyright $\odot 2015$ the authors $\quad 0270-6474 / 15 / 357414-14 \$ 15.00 / 0$ centration. These cotransporters are especially relevant during developmental stages when GABA is excitatory and throughout life to maintain inhibitory control of GABAergic synapses. The $\mathrm{Na}-\mathrm{K}-2 \mathrm{Cl}$ type 1 cotransporter (NKCC1) is mainly expressed during early stages of development in neurons and contributes to maintain high intracellular chloride concentration $\left(\left[\mathrm{Cl}^{-}\right]_{\mathrm{i}}\right)$. Under increased $\left[\mathrm{Cl}^{-}\right]_{\mathrm{i}}, \mathrm{GABA}_{\mathrm{A}}$ receptor activation results in $\mathrm{Cl}^{-}$ efflux that consequently depolarizes the neuron (Ben-Ari et al., 2007, 2012). However, NKCC1 expression in sensory neurons is increased in adulthood and mediates normal sensory processing mechanisms, such as primary afferent depolarization or presynaptic inhibition (Price et al., 2009). After peripheral nerve injury, NKCC1 increases in sensory neurons contributing to the anterograde and retrograde propagation of electrical activity (Cervero et al., 2003) and, importantly, promoting the regeneration of sensory axons (Pieraut et al., 2007) upon interleukin-6 (IL-6) regulation (Pieraut et al., 2011). However, although increased expression of NKCC1 after peripheral nerve injury seems to be a relevant feature for the regeneration of sensory axons, how its increased activity modulates regeneration and whether or not this mechanism is selective of a DRG subpopulation have not yet been investigated.

The regulation of mitogen-activated protein kinase (MAPKs) signaling cascades, such as extracellular signal-regulated protein kinases (ERKs; Napoli et al., 2012), p38 MAPKs (Raman et al., 2007), and c-Jun N-terminal protein kinases (JNKs; Lindwall and Kanje, 2005), has also been linked to pro-regenerative processes 
of axonal growth (Zrouri et al., 2004; Perlson et al., 2005). Despite the role of $\mathrm{Na}^{+}$(Wolf et al., 2001) and $\mathrm{Cl}^{-}$(André et al., 2003) in the increase of calcium levels (reviewed in Waxman and Zamponi, 2014) that modulates the activation of MAPK signaling (Wolf et al., 2001; Mandolesi et al., 2004), the question of how changes in cation-chloride cotransporters may affect the expression of MAPKs has not been studied. Therefore, in the present study we assessed the role of NKCC1 in the regeneration of different types of sensory neurons and its modulation of different MAPK signaling pathways after nerve injury.

\section{Materials and Methods}

Animals. A total of 41 adult female Sprague Dawley rats (weight, $240 \pm$ $30 \mathrm{~g}$ ) was used in the experiments. Further, 6 pups at postnatal day 21 (P21; for dissociated DRG cultures) and 18 pups at P7 (for DRG organotypic cultures) were also used. Adult animals were housed in standard cages and kept on standard laboratory food and water ad libitum with a $12 \mathrm{~h}$ light/dark cycle. All of the experimental procedures were approved by the Ethics Committee of the Universitat Autonoma de Barcelona and followed the guidelines of the European Commission on Animal Care (EU Directive 2010/63/EU).

Dissociated DRG cultures. Poly-D-lysine was added to Petri dishes at 10 $\mu \mathrm{l} / \mathrm{ml}$ for $2 \mathrm{~h}$ at $37^{\circ} \mathrm{C}$, dried, and further coated for $2 \mathrm{~h}$ with $1 \mu \mathrm{l} / \mathrm{ml}$ laminin. DRGs were harvested from P21 Sprague Dawley rats and placed in Gey's salt solution (Sigma-Aldrich) with $6 \mathrm{mg} / \mathrm{ml}$ glucose. After peeling the DRGs from connective tissue, samples were dissociated in $10 \%$ collagenase, $10 \%$ trypsin, and 10\% DNase in HBSS without Ca-Mg for 30 $\mathrm{min}$ at $37^{\circ} \mathrm{C}$, with shaking every $10 \mathrm{~min}$. Cells were resuspended in adult Neurobasal A medium (Life Technologies) supplemented with $1 \times$ B-27, $1 \times$ penicillin/streptomycin, $1 \mathrm{~mm}$ L-glutamine, and $6 \mathrm{mg} / \mathrm{ml}$ glucose (Sigma-Aldrich); and incubated for $24 \mathrm{~h}$.

Bumetanide (Sigma-Aldrich) suspended in dimethylsulfoxide (DMSO) at 10, 50100 , and $200 \mu \mathrm{m}$; furosemide (Sigma-Aldrich) at 50, 100, and 200 $\mu \mathrm{M}$; or only vehicle (DMSO) was added to the medium. After $72 \mathrm{~h}$ of incubation, $0.15 \mathrm{mg} / \mathrm{ml} \mathrm{MTT} \mathrm{(Sigma-Aldrich)} \mathrm{was} \mathrm{added,} \mathrm{and} \mathrm{the} \mathrm{cells}$ were further incubated for $2 \mathrm{~h}$ at $37^{\circ} \mathrm{C}$. The formazan crystals were dissolved in $200 \mu \mathrm{l}$ of DMSO, and $150 \mu \mathrm{l}$ was transferred to 96-well plates. The optimal density was determined with a microculture plate reader (BioTek) at 570 and $620 \mathrm{~nm}$ (to counteract the noise of the plastic) and analyzed with KcJunior software.

For neurite outgrowth analysis, images were collected using a PlanApochromat $10 \times$ objective, and Image $(\mathrm{NIH}$, Bethesda) was used for measuring the neuronal soma diameter and neurites. Neurons that extended neurites (primary neurite) longer than two cell body diameters were counted. Measurements were performed from at least three wells per rat, with three rats per experimental condition.

$D R G$ explant and neurite outgrowth analyses. A volume of $450 \mu \mathrm{l}$ of rat tail type I collagen solution (BD Biosciences) at a concentration of 3.4 $\mathrm{mg} / \mathrm{ml}$ was mixed with $50 \mu \mathrm{l}$ of $10 \times$ basal Eagle's medium (Invitrogen) and $2 \mu \mathrm{l}$ of $7.5 \%$ sodium bicarbonate solution, and DMSO or bumetanide suspended in DMSO was added at two different concentrations, 100 and $200 \mu \mathrm{M}$. Single drops of $30 \mu \mathrm{l}$ were deposited on poly-D-lysine ( $1 \mu \mathrm{g} / \mathrm{ml}$, Sigma-Aldrich)-coated coverslips, which were kept in the incubator at $37^{\circ} \mathrm{C}$ and $5 \% \mathrm{CO}_{2}$ for $2 \mathrm{~h}$ to induce collagen gel formation.

Sprague Dawley rats of postnatal day 7 were decapitated, and lumbar DRGs were dissected, placed in cold Gey's balanced salt solution (SigmaAldrich), enriched with $6 \mathrm{mg} / \mathrm{ml}$ glucose, and cleaned from blood and meningeal debris. DRG explants were then placed on gelled collagen droplets and covered by a second drop of $30 \mu \mathrm{l}$ of collagen type I solution (Allodi et al., 2011). The embedded samples were placed in the incubator for $45 \mathrm{~min}$ before adding Neurobasal medium (Invitrogen), supplemented with B27 (Life Technologies), glutamine, and penicillin/streptomycin (Sigma-Aldrich). After $1 \mathrm{~d}$ in culture, the medium was removed and changed with a penicillin/streptomycin-free medium, and the DRG explants were cultured for 2 more days. To quantify neurites outgrowth, images collected using a Plan-Apochromat $10 \times$ objective were measured using Neurite-J java plug-in for ImageJ. This software allows fast semi- automatic neurite outgrowth analysis adapting Sholl methods to organotypic cultures (Torres-Espín et al., 2014).

In vivo nerve injury and drug administration. Adult female Sprague Dawley rats were anesthetized by injection of ketamine $(10 \mathrm{mg} / \mathrm{kg}$, i.p.; Imalgene 500, Rhône-Merieux) and xylazine ( $1 \mathrm{mg} / \mathrm{kg}$; Rompun, Bayer). The right sciatic nerve was exposed at the mid-thigh, transected at $92 \mathrm{~mm}$ from the tip of the third toe, and repaired by epineurial sutures (10-0), maintaining the fascicular alignment of tibial, peroneal, and sural branches. Then, the wound was sutured in planes and disinfected. The animals were left to recover on a warm pad and returned to their home cages.

Bumetanide was dissolved in saline and 1\% DMSO. Two groups of rats were administered bumetanide at $30 \mathrm{mg} / \mathrm{kg}(n=7)$ or vehicle $(n=7)$ by intraperitoneal injection daily from 1 until $16 \mathrm{~d}$ postinjury (DPI).

Retrograde labeling and neuronal counting. Eight of the 41 adult rats were kept for 10 DPI to assess axonal regeneration in the short term. Then, the sciatic nerve was transected $14 \mathrm{~mm}$ distal to the suture site and labeled with Fluoro-Gold (FG; Fluorochrome) to identify motor and sensory neurons that had regenerated their axons to the sectioned point. Back-labeling with FG was performed by exposing the tip of the severed nerve to $5 \%$ FG in distilled water for $1 \mathrm{~h}$ in a vaseline well. Leakage was prevented and, after retrieval of the vaseline well, the area was rinsed with saline to eliminate any excess of the retrotracer. The wound was sutured, and the rats were kept for $7 \mathrm{~d}$ to allow the accumulation of the tracer in the soma of spinal motoneurons and DRG sensory neurons. Then, the rats were deeply anesthetized and transcardially perfused with $4 \%$ paraformaldehyde in PBS. A lumbar L4-L6 spinal cord segment and L4-L6 DRGs were removed, postfixed, and transferred to $30 \%$ sucrose in PBS. Transverse sections were cut at a thickness of $40 \mu \mathrm{m}$ for spinal cord and $20 \mu \mathrm{m}$ for DRGs with a cryotome (Leica). Sections were viewed under an epifluorescence microscope (BX51, Olympus) to visualize FG-labeled neurons. Using the fractionator principle (Gundersen, 1986), all retrogradely labeled motoneurons with a visible cell nucleus in the sections and all retrogradely labeled sensory neurons with a visible cell nucleus in the sections were counted.

Electrophysiological assessment of nerve regeneration. Sciatic nerve conduction tests were performed before surgery and repeated at different days after injury. Under pentobarbital anesthesia (40 mg/kg, i.p.), the sciatic nerve was stimulated at the sciatic notch with single electrical pulses. Compound nerve action potentials (CNAPs) were recorded by means of monopolar needles ( $28 \mathrm{ga}$ ), and the active electrode was inserted near the lateral plantar nerve and the reference at the fourth toe, with a ground needle placed at the knee. The CNAPs were displayed in an oscilloscope (Sapphyre 4M, Medelec Vickers). The amplitude of the CNAP was measured in microvolts and expressed as a percentage with respect to values obtained before surgery from the hindlimb of each rat, to evaluate the regeneration of large myelinated nerve fibers (Navarro and Udina, 2009). During the tests, the rat body temperature was maintained by means of a warming flat pad that contained a thermostat.

Assessment of skin nociceptive reinnervation. The progression of nociceptive reinnervation of the hindpaw was assessed by means of the pinprick test at different times after injury (Navarro et al., 1994; Cobianchi et al., 2014). Animals were gently kept in a cloth with the sole of the injured paw facing upward, and the skin was stimulated with a needle progressively from proximal to distal at specific sites of the following two different regions of the hindpaw plantar surface: the lateral (normally innervated by sciatic nerve), and the medial (innervated by saphenous nerve; Cobianchi et al., 2013, 2014). Responses were recorded as positive only when clear pain reaction (as fast withdrawal and vocalization) was triggered by the stimulation. Positive responses were taken as sign of skin functional reinnervation. A composite score was calculated as the mean number of responses per group at each day of testing.

Sensory threshold measurement. Sensibility to mechanical stimuli was measured with an electronic Von Frey algesimeter (Bioseb) before surgery and at different times after injury. Rats were placed on a wire net platform in plastic chambers $15 \mathrm{~min}$ before the experiment for habituation. The Von Frey probe was pressed against the plantar surface of the hindpaw at two specific lateral and medial test sites (Cobianchi et al., 2014). The mechanical nociceptive threshold was taken as the mean of three measurements per test site, with a 5 min interval between each 
measurement, and expressed as the pressure (in grams) at which rats withdrew their paws in response to the stimulus. A cutoff force was set to $40 \mathrm{~g}$, at which time the probe lifted the paw without response. The contralateral paw of each rat was tested as control each testing day.

Protein extraction and Western blot. Total proteins from culture explants or lumbar L4-L5 DRGs were homogenized in a RIPA buffer (containing $50 \mathrm{~mm}$ Tris- $\mathrm{HCl}, \mathrm{pH} 7.5,1 \%$ Triton $\mathrm{X}-100,0.5 \%$ sodium deoxycholate, $0.2 \%$ SDS, $100 \mathrm{~mm} \mathrm{NaCl}$, and $1 \mathrm{~mm}$ EDTA), adding $10 \mu \mathrm{l} / \mathrm{ml}$ protease inhibitor cocktail (Sigma-Aldrich) and PhosSTOP phosphatase inhibitor cocktail (Roche). After clearance, protein concentration was measured by Lowry assay (DC Protein Assay, Bio-Rad). Twenty micrograms of protein of each sample were loaded in SDS-polyacrylamide gels. The transfer buffer was $25 \mathrm{~mm}$ Trizma Base, $192 \mathrm{~mm}$ glycine, and 20\% $(\mathrm{v} / \mathrm{v})$ methanol, $\mathrm{pH}$ 8.4. The membranes were blocked with 5\% BSA in PBS plus $0.1 \%$ Tween-20 for $1 \mathrm{~h}$, and then incubated with primary antibodies at $4^{\circ} \mathrm{C}$ overnight. The primary antibodies used were as follows: mouse anti-GAPDH (1:20,000; Merck Millipore), mouse anti-NKCC1 (1:1000; Developmental Studies Hybridoma Bank), sheep anti-phospho (p)-NKCC1 (1:1000; purchased from the Protein Phosphorylation and Ubiquitylation Unit, University of Dundee, UK), phosphoJNK1/2 (1: 500; Merck Millipore), p38 MAPK (1:1000; Cell Signaling Technology), and phospho-ERK1/2 (1:500; Cell Signaling Technology). Horseradish peroxidase-coupled secondary antibody (1:5000; Vector Laboratories) incubation was performed for $1 \mathrm{~h}$ at room temperature. The membranes were visualized using an enhanced chemiluminescence method, and the images were collected and analyzed with a Gene Genome apparatus, using Gene Snap and Gene Tools software (Syngene).

Assessment of phosphorylated ERK1/2, p38 MAPK, and JNK1/2/3 by ELISA. Protein homogenates extracted from organotypic cultures and from DRGs of animals treated with vehicle or bumetanide at 16 DPI were used for the assessment of the phosphorylated state of ERK1/2 (pT202/ Y204), p38 MAPK (pT180/Y182), and JNK1/2/3 (pT183/Y185) using an ELISA kit (Abcam). Quantification was performed with at least three samples for each condition. Fifty microliters of the protein lysate were added to each well of the PhosphoTracer microplate ( $20 \mu \mathrm{g}$ of protein). A further $50 \mu \mathrm{l}$ of the antibody mix (Capture antibody reagent plus detection antibody) were added to each well and was incubated for $1 \mathrm{~h}$ at room temperature on a microplate shaker at $300 \mathrm{rpm}$. After washing wells with PBS-Tween $0.05 \%$, the substrate mix [developer solution: 10acetyl-3-7-dihydroxyphenoxazine (ADHP) and ADHP Dilution Buffer] was added to each sample well while shaking for $10 \mathrm{~min}$. Signal ELISA quantification was performed using photodetection at 590-600 nm bandwidths (BioTek).

Immunohistochemical labeling. Dissociated neurons and DRG explant cultures were fixed in 4\% paraformaldehyde in PBS for $20 \mathrm{~min}$ and blocked with 1.5\% normal donkey antiserum (Merck Millipore). Samples were then incubated for $2 \mathrm{~h}$ (DRG-dissociated cultures) or $48 \mathrm{~h}$ (DRG explants) with the following primary antibodies: chicken antineurofilament heavy chain [200 kDa neurofilament (NF200; 1:1000; Merck Millipore], mouse anti- $\beta$-III-tubulin ( $\beta$-Tub; $1: 1000$; Covance), and anti-NKCC1 (1:500; Developmental Studies Hybridoma Bank). After washing, the sections were incubated overnight (DRG explants) or for $1 \mathrm{~h}$ (dissociated cells) with secondary antibodies.

For the in vivo localization study, rats were perfused, and spinal cord and DRG samples were processed as indicated above. For immunohistochemical labeling, sections were blocked with PBS-Triton $0.3 \%$ and normal donkey serum $5 \%$, and were incubated overnight at $4^{\circ} \mathrm{C}$ with the following primary antibodies: chicken anti-NF200, mouse anti- $\beta$-Tub, goat anti-calcitonin gene-related peptide (CGRP; 1:200; Abcam), antiGriffonia simplicifolia lectin 1 (IB4; Vector Laboratories), anti-neuropeptide Y (NPY; 1:600; Immunostar), anti-NKCC1 (1:500), rabbit anti-parvalbumin (Parv; 1:500; Swant), anti-mouse GFAP (1:500; Abcam), anti-rabbit JNK (1:200; Millipore), anti-rabbit pERK (1:200; Cell Signaling Technology). After washes, sections were incubated for $1 \mathrm{~h}$ at room temperature with Alexa Fluor 488- or Alexa Fluor 594-conjugated secondary antibodies (1:200; Life Technologies).

For assessing skin reinnervation, plantar pads corresponding to the lateral side were removed at the end of the functional follow-up, $60 \mathrm{~d}$ after sciatic nerve injury. Cryotome sections $60 \mu \mathrm{m}$ thick were processed for colabeling against protein gene product (PGP) 9.5 (rabbit; 1:1000; UltraClone, Lucigen), a pan-neuronal marker, and NF200, a marker of myelinated fibers (Navarro et al., 1997). Secondary antibodies were conjugated to $\mathrm{Cy} 3$ and Cy5. The images were viewed under confocal microscopy to visualize immunoreactive nerve fibers that had arrived at the subepidermal plexus and the epidermis. To quantify the amount of innervation at the dermis, the integrated density of immunolabeling of PGP9.5 and NF200 was calculated in $20 \times$ images using Image J software. The number of intraepidermal nerve fibers (IENFs) was counted in a 1-mm-long segment of the footpad epidermis (Cobianchi et al., 2014). Three sections per animal were used for these measurements, and values were averaged.

Statistical analysis. Data are presented as the mean \pm SEM. Statistical analysis of nociceptive thresholds was made by two-way ANOVA with group and time after injury as factors, followed by Bonferroni post hoc comparisons. Statistical significance for immunoblotting data was calculated by one-way and two-way ANOVA (for multiple-groups comparison) followed by Tukey's post hoc test when necessary. The level of statistical significance was $5 \%(p<0.05)$ in all of the analyses.

\section{Results \\ Phosphorylation of NKCC1 in DRG is increased after peripheral nerve injury}

To corroborate that NKCC1 activity increases after peripheral nerve injury, we analyzed the levels of NKCC1 and pNKCC1 by Western blot (WB) and immunohistochemistry. As previously reported (Mòdol et al., 2014), there were no significant changes in the expression of NKCC1 in injured DRGs compared with control (naive) samples or with the contralateral (uninjured) side (Fig. 1A). In contrast, the analysis of pNKCC1 expression showed differences depending on the time postinjury that was analyzed (two-way ANOVA, $p<0.01$; Fig. $1 A$ ). A progressive increase in pNKCC1 expression after sciatic nerve injury was observed in the injured DRGs compared with the uninjured DRGs at 3 DPI $(p<0.05)$, 8 DPI $(p<0.01)$, and 16 DPI $(p<0.001)$. Significant differences were also found when comparing injured rats at $8 \mathrm{DPI}(p<0.05)$ and $16 \mathrm{DPI}(p<0.001)$ to control intact rats (Fig. $1 A, B$ ). Immunohistochemical labeling showed that NKCC1 localization changed from the cytoplasm to the membrane of DRG neurons after axotomy (Fig. 1C), corroborating the increase in pNKCC1 (active state) after the injury observed by immunoblotting.

We also found that NKCC1 blocking by bumetanide administration modified the pNKCC1 increase in vivo (at 16 DPI) by Western blot and immunohistochemistry. Our results indicate that the increase of pNKCC1 expression in DRGs is prevented in bumetanide-administered animals compared with the vehicleadministered animals after nerve injury $(p<0.001$; Fig. $1 D, E)$. This change is also reflected by the prevention of NKCC1 membrane insertion (Fig. 1F).

\section{NKCC1 is expressed in sensory neurons before and after nerve injury}

To investigate whether NKCC1 is differentially expressed in DRG neuronal subpopulations, we performed an immunohistochemical colocalization study of NKCC1 with several markers of DRG neurons before and after sciatic nerve injury. The following neuronal markers were used: phosphorylated NF200 for mediumand large-diameter neurons with myelinated axons, IB4 for small nonpeptidergic neurons, and CGRP for small- to medium-size peptidergic neurons (Michael et al., 1997). Results of colocalization between NKCC1 and CGRP showed a tendency to decrease the percentage of CGRP+/NKCC1+ neurons at 16 DPI $(35 \%$ uninjured vs $18 \%$ injured; $p=0.07$; Fig. $2 A-C)$. In contrast, the 
A

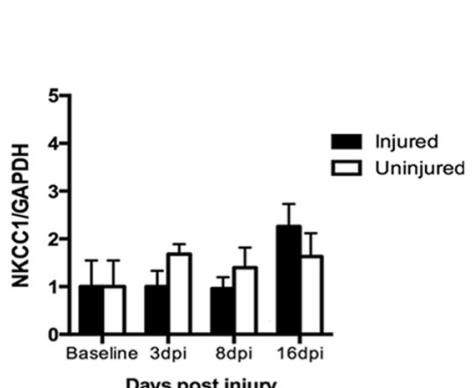

B

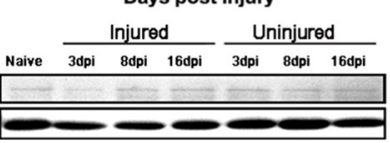

PNKCC1 GAPDH

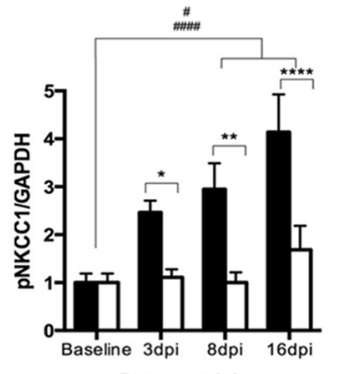

Days post injury
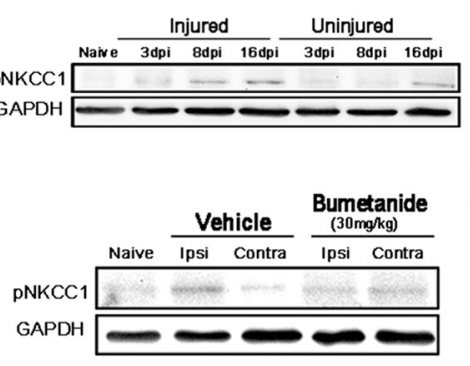

$\mathbf{E}$
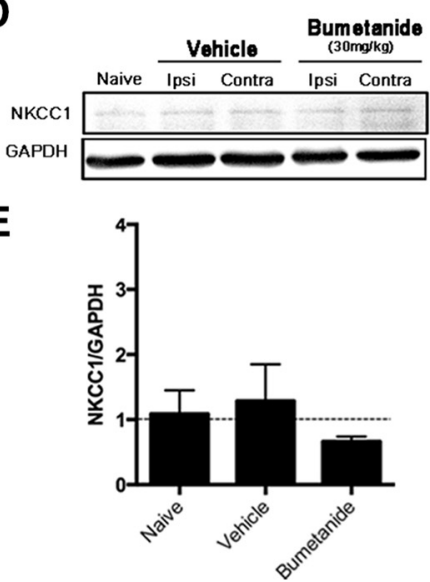

C

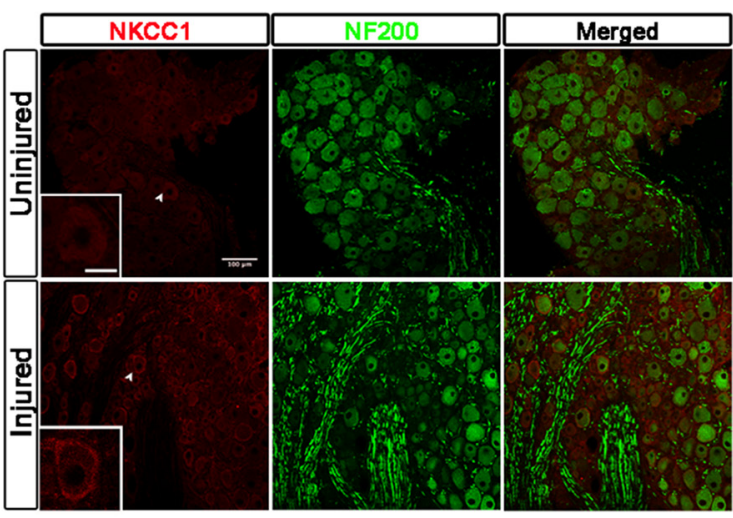

$\mathbf{F}$

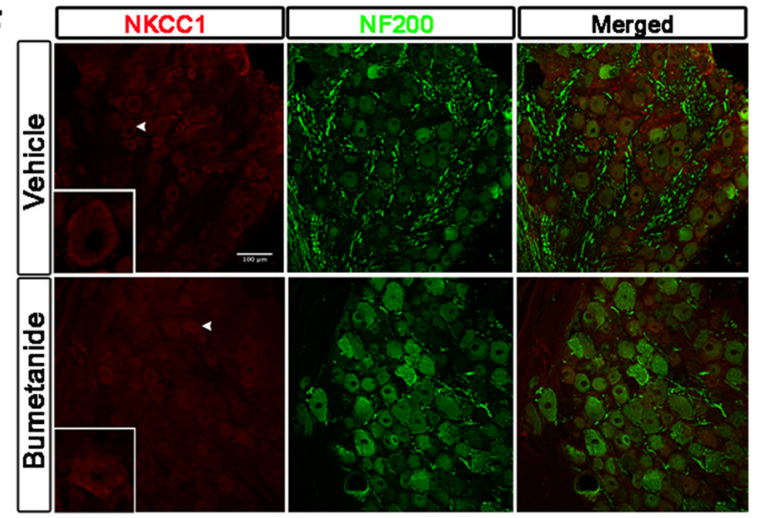

Figure 1. pNKCC1 expression is increased after nerve injury in DRGs. $A, B$, Representative WBs and quantification of NKCC 1 and pNKCC 1 expression changes at early time points (3, 8, and 16 DPI) in injured (black) and uninjured (white) samples. $\boldsymbol{D}, \boldsymbol{E}$, Increased expression of pNKCC1 after injury is prevented by bumetanide administration $\left(30 \mathrm{mg} / \mathrm{kg}\right.$; vehicle vs naive, ${ }^{* *} p<0.01$; vehicle vs bumetanide ${ }^{* * *} p<0.001$ ) in the ipsilateral (ipsi) and contralateral (contra) side of the injured paw. WB quantification is shown at the final time point of bumetanide administration (16 DPI). $\boldsymbol{C}, \boldsymbol{F}$, Confocal images show the increased localization of NKCC1 in the membrane of DRG neurons, labeled with NF200 structural marker, in injured animals and its prevention by bumetanide administration. Arrowheads point membrane-bound or cytoplasmatic localization of NKCC1 in injured $(\boldsymbol{C})$ or vehicle and bumetanide treated groups $(\boldsymbol{F})$. Scale bar, $100 \mu \mathrm{m}$. Inset boxes show detailed neurons labeled with NKCC1 (scale bar, $10 \mu \mathrm{m}$ ). Data are presented as the mean \pm SEM. Ipsi, Ipsilateral; Contra, contralateral.

percentage of IB4 + /NKCC1 + neurons showed a slight, nonsignificant increase after injury (19\% uninjured vs 30\% injured; Fig. 2D-F). These results also indicate that the percentage of $\mathrm{CGRP}+/ \mathrm{NKCC} 1+$ neurons is higher than that of IB4+/NKCC1+ neurons in uninjured DRGs, whereas the balance is reversed after injury. However, the fact that both markers experience changes in expression after axotomy in regenerating neurons cannot be discarded. NPY was barely detected in a few control DRG neurons, but its expression markedly increased after sciatic nerve section in L5 DRG neurons, being detected in about half of the NKCC1+ neurons (Fig. 2K). Additionally, colabeling of NF200+ and NKCC $1+$ showed that $\sim 50 \%$ of DRG neurons in both uninjured and injured animals express NKCC1 (52\% uninjured vs 51\% injured; $p=$ n.s.), further indicating that $\mathrm{NKCC} 1$ expression does not change in myelinated neurons after the injury (Fig. 2G-I). Together, the present results indicate that NKCC1 expression is heterogeneous, and is present in both nonmyelinated and myelinated fiber neuronal populations before and after peripheral nerve injury (Fig. $2 K$ ). Further, we performed a morphometric analysis of NKCC1 + neurons in injured and uninjured animals to avoid changes in subpopulation markers induced by the injury. The frequency distribution showed no differences in soma sizes of DRG neurons that express NKCC1 before and after injury $\left(p=0.33, \chi^{2}\right.$ test $)$.

\section{Blocking NKCC1 prevents outgrowth of myelinated but not unmyelinated neurites}

As reported by Pieraut et al. (2007, 2011), the increased NKCC1 activity in DRG neuronal soma after nerve injury plays a role in the regeneration of sensory fibers. Thereby, to determine whether increased NKCC1 activity after injury is necessary for the growth of myelinated and nonmyelinated axons, we used two different in vitro models and assessed the effects of bumetanide (a NKCC1 activity blocker). In dissociated DRG neuron cultures, blocking NKCC1 activity by bumetanide addition impaired the outgrowth of NF200-labeled neurites in a dose-dependent manner $(p<$ 0.001, one-way ANOVA; Fig. $3 A-C$ ). Indeed, all of the doses tested showed significant differences compared with vehicleadded cultures ( $p<0.05$ and $p<0.001$, respectively). Further, we verified the NF200 staining by measuring the size of labeled neurons soma. Our results indicate that soma size was similar for all the administered bumetanide concentrations and the vehicle (DMSO)-administered cultures (Fig. 3E). Morphometric analysis shows no differences regarding the soma size distribution of NF200-labeled neurons (Fig. 3D). MTT assay in cultures treated with DMSO or bumetanide at several concentrations (10,50, and $100 \mu \mathrm{M})$ showed that none of the doses administered affected neuronal survival ( $p=$ n.s., one-way ANOVA; data not shown), supporting the role of NKCC1 activity in neuronal outgrowth. 


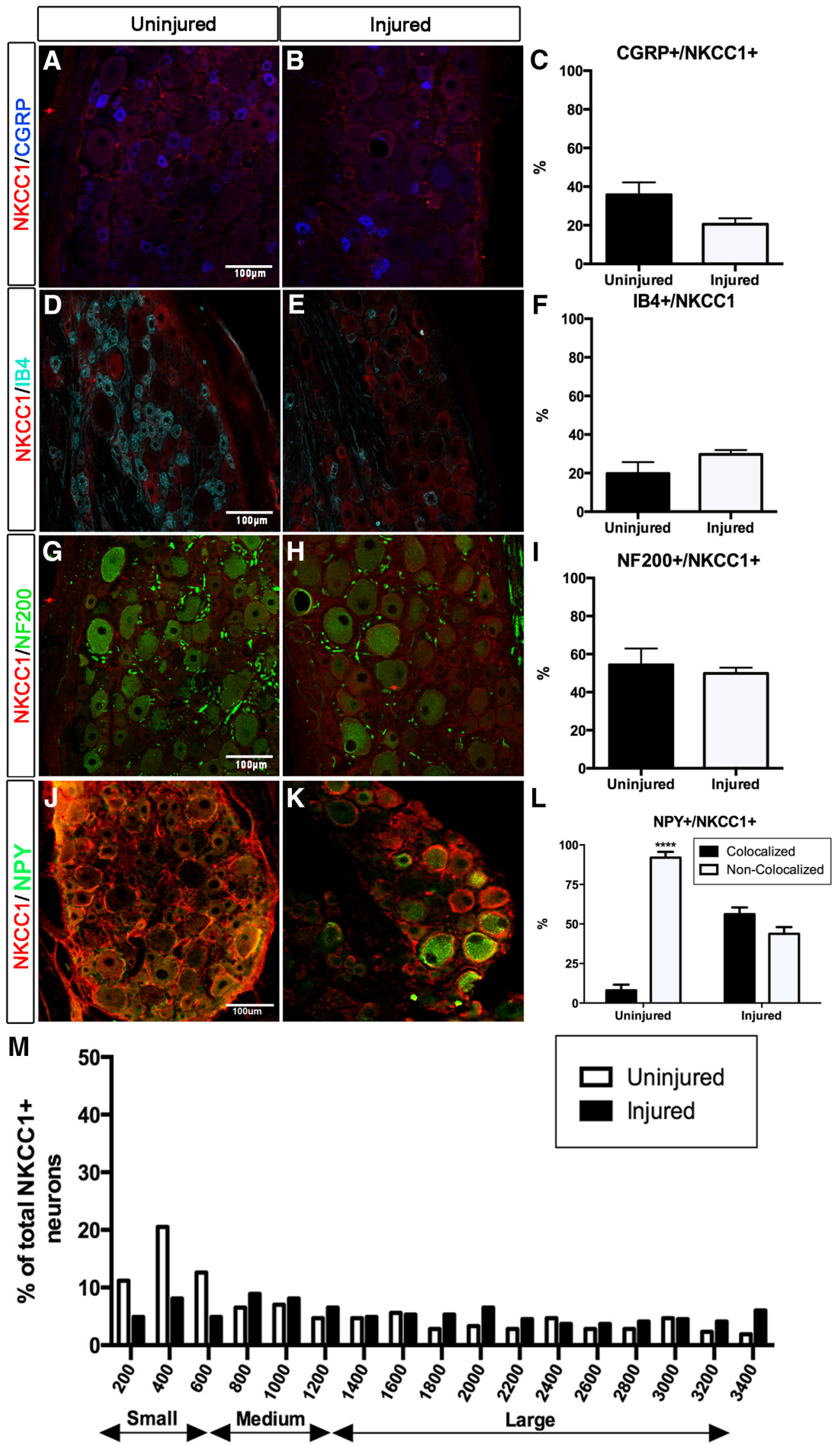

Cross Sectional Area $\left(\mu \mathrm{m}^{2)}\right.$ 

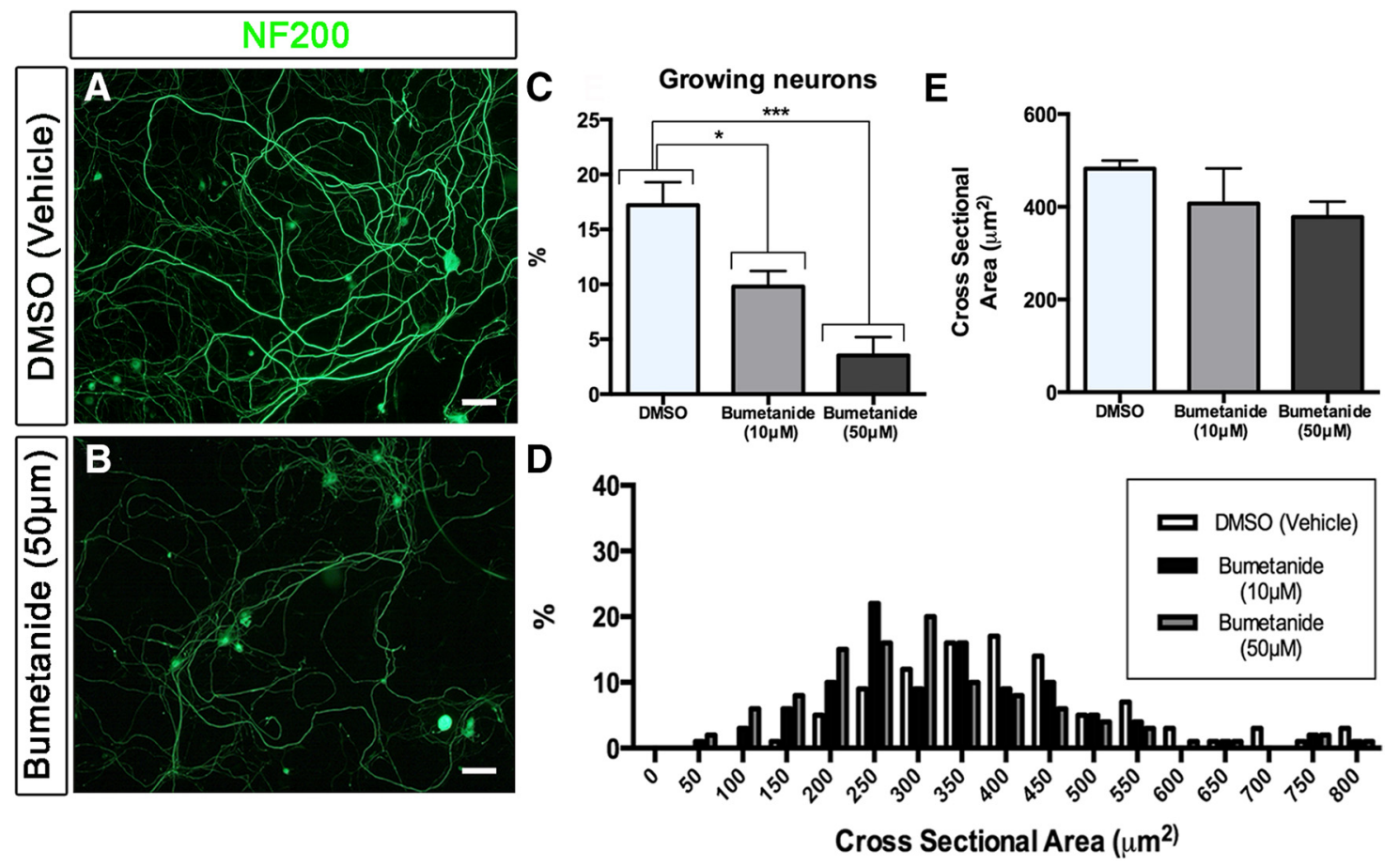

Figure 3. Blocking NKCC1 activation with bumetanide prevented neurite outgrowth of NF200+ (myelinated) neurons. $\boldsymbol{A}-\boldsymbol{C}$, Confocal images of DRG-dissociated cultures ( $\boldsymbol{A}, \boldsymbol{B})$ and quantification of neurite outgrowth $(\boldsymbol{C})$ in vehicle-administered (DMSO) or bumetanide-administered cultures. Bumetanide administration induced a decrease in the amount of neurite outgrowth at both concentrations tested (10 and $50 \mu \mathrm{M})$ compared with the vehicle $\left({ }^{*} p<0.05\right.$ and ${ }^{* * *} p<0.01$, respectively). $\boldsymbol{D}$, Morphometric analysis of the size of NF200+ neuronal soma and $(\boldsymbol{E})$ mean soma size for each administered group of cultures. Scale bar, $100 \mu \mathrm{m}$. Data are presented as the mean \pm SEM.

To further investigate the role of NKCC1 on axonal regeneration, we then tested whether NKCC1 blocking affects neurite outgrowth in organotypic cultures, since this type of culture maintains a cell environment that is closer to the in vivo situation (Allodi et al., 2011). In this model, a 3D collagen matrix surrounds DRGs, preventing the "full contact" of DRGs with the administered drug. For this reason, the concentration of bumetanide was increased to 100 and $200 \mu \mathrm{M}$. The results of DRG cultures corroborated that the outgrowth of myelinated fibers $(\mathrm{NF} 200+)$ was reduced by bumetanide administration at high concentration $(200 \mu \mathrm{M})$ compared with vehicle (DMSO)administered explants $(p<0.0001$; Fig. $4 A-C)$. We also tested whether bumetanide differentially affects neurite outgrowth of myelinated versus nonmyelinated fibers. For this purpose, we used staining of $\beta$-Tub, a microtubule-associated protein that labels all types of sensory neurons. The analysis of $\beta$-Tub-positive neurites showed a slight tendency to decrease under bumetanide administration to the DRG culture ( $p=0.07$; Fig. $4 D-F)$. To isolate the outgrowth of nonmyelinated fibers, we focused on the NF200 $-/ \beta$-Tub + neurites. In cultures treated with bumetanide $\mathrm{NF} 200-/ \beta-\mathrm{Tub}+$, neurites increased in areas proximal to the soma compared with vehicle-treated cultures $(p<0.001$; Fig.

\section{$\leftarrow$}

Figure 2. Immunohistochemical analyses of NKCC 1 expression in DRG neuron subpopulations in injured and uninjured animals. $\boldsymbol{A}, \boldsymbol{B}, \boldsymbol{D}, \boldsymbol{E}, \boldsymbol{G}, \boldsymbol{H}, \boldsymbol{J}, \boldsymbol{K}$, Confocal images of unmyelinated peptidergic (CGRP $+; \boldsymbol{A}, \boldsymbol{B})$, nonpeptidergic (IB4+; $\boldsymbol{D}, \boldsymbol{E})$, myelinated (NF200+; $\boldsymbol{G}, \boldsymbol{H})$, and $\mathrm{NPY}+(\boldsymbol{J}, \boldsymbol{K})$ DRG neurons expressing NKCC1 in injured and uninjured rats. Scale bar, $100 \mu \mathrm{m} . \boldsymbol{C}$, $F, I, L$, There were no significant differences in the quantification of NKCCI expression between injured and uninjured animals $(\boldsymbol{C}, \boldsymbol{F}, \boldsymbol{I})$, except for the coexpression of NPY $(\boldsymbol{L})$. Data are presented as the mean \pm SEM. J, Morphometric analysis of NKCC 1 + neurons in uninjured (white) and injured (black) DRGs. $M$, The frequency distribution of soma diameter of neurons expressing NKCC 1 shows no differences between uninjured and injured animals.
$4 M)$. Additionally, to corroborate the effect observed by bumetanide administration we also tested the effect of another NKCC1 blocker (furosemide) on myelinated fibers growth. To our knowledge, furosemide has not been used in DRG organotypic cultures previously. Interestingly, our results indicate the same effect as observed with bumetanide (i.e., furosemide reduced neurite outgrowth in a dose-dependent manner; Fig. $4 G-I$ ) without affecting $\beta$-Tub-positive neurites (Fig. $4 J-L, N$ ). These results indicate that blocking NKCC1 activity promoted neurite outgrowth of nonmyelinated fibers but decreased that of myelinated fibers.

\section{Bumetanide administration decreases NF200+ neuron} regeneration in vivo after nerve injury

As in vitro results showed a specific decrease in the amount of neurite outgrowth of NF200+ DRG neurons, we then blocked NKCC1 activity in animals with sciatic nerve injury to elucidate the effect in vivo. For that purpose, we performed a sciatic nerve section and suture repair, and labeled with FG to observe whether regeneration (retrolabeled neurons in DRGs) was affected by bumetanide administration (from 1 to $16 \mathrm{DPI}$ ). We checked whether neurons labeled with FG were also positive for GAP-43, a protein that is upregulated in regenerating neurons (Rossi et al., 2007), and whether or not these neurons also expressed NKCC1 in the plasma membrane. Our results indicate that all the neurons labeled with FG were indeed positive for GAP-43 and also expressed NKCC1 in the cell membrane (Fig. 5).

We then analyzed whether bumetanide administration influenced axonal regeneration of DRG neurons by assessing the number of cells labeled after the application of FG in the injured nerve at 16 DPI. Immunohistochemical colabeling with $\beta$-Tub was performed to estimate the total number of neurons. Results showed a decrease in the percentage of FG + neurons in 


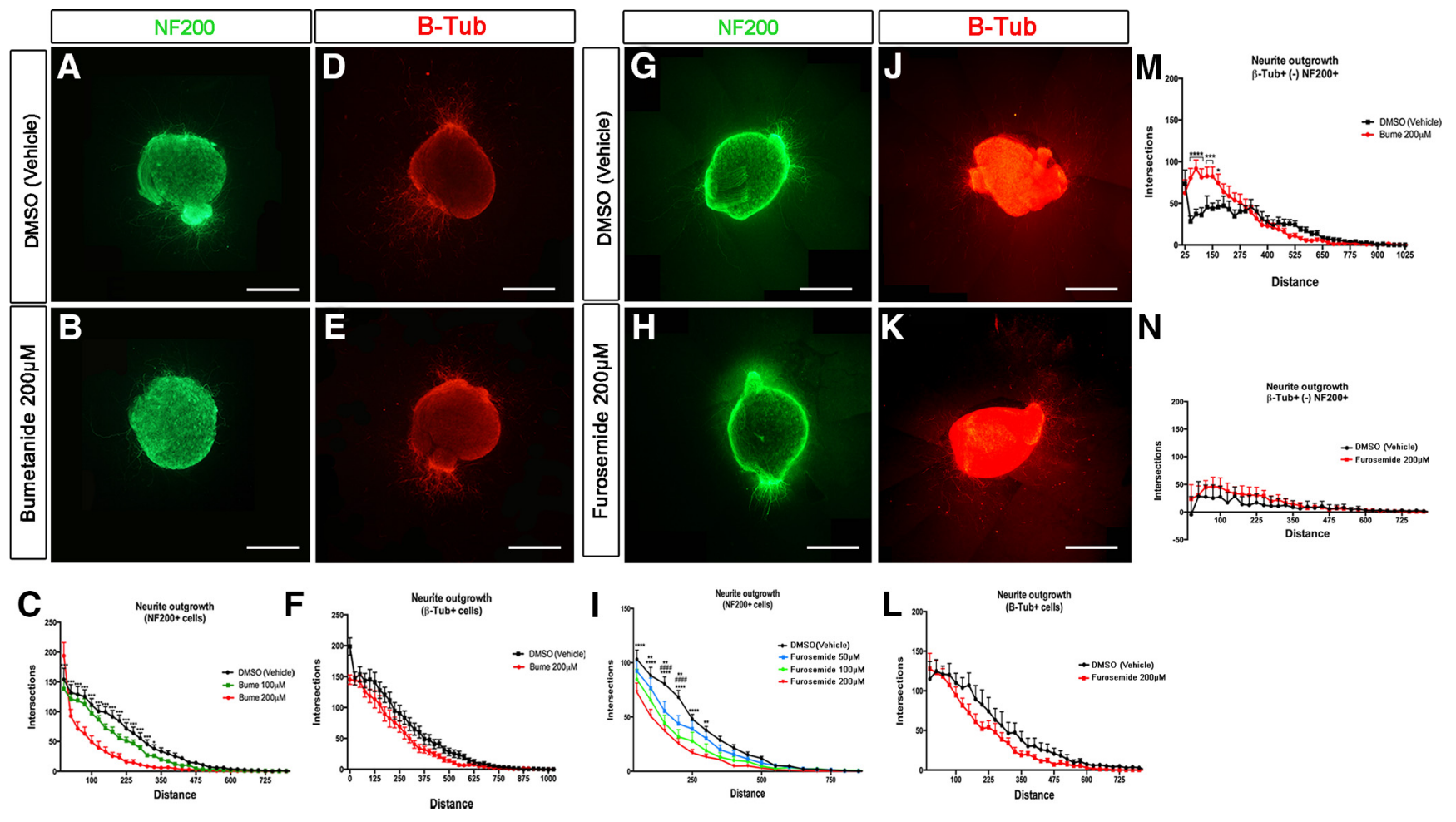

Figure 4. Blocking NKCC 1 activation with bumetanide or furosemide prevented neurite outgrowth of NF200+ (myelinated) neurons. $A, B, D, E, G, H, J, K$, Images of DRG explants labeled with NF200 $(\boldsymbol{A}, \boldsymbol{B}, \boldsymbol{G}, \boldsymbol{H})$ or $\beta$-Tub $(\boldsymbol{D}, \boldsymbol{E}, \boldsymbol{J}, \boldsymbol{K}) . \boldsymbol{C}, \boldsymbol{F}, \boldsymbol{I}, \boldsymbol{L}$, Bumetanide or furosemide administration reduced neurite outgrowth as shown by the decrease in the number of intersections in NF200 + -labeled explants $\left({ }^{* * *} p<0.005\right.$ and ${ }^{*} p<0.05$, DMSO (vehicle) vs bumetanide $200 \mu \mathrm{m}$; ${ }^{* *} p<0.01$, DMSO vs furosemide $50 \mu \mathrm{m}$; \#\#\#p $<0.0001$ and \#\#\#p $<0.001$, DMSO vs furosemide $100 \mu \mathrm{M}$; ${ }^{* * * *} p<0.0001$ and ${ }^{* *} p<0.01$, DMSO vs furosemide $200 \mu \mathrm{m}$; (and $\boldsymbol{I}$, respectively), but not in those labeled with $\beta$-Tub ( $\boldsymbol{F}$ and $\boldsymbol{L}$, respectively). $\boldsymbol{M}, \boldsymbol{N}$, Plots of the neurites that were $\beta$-Tub + but NF200-, corresponding to unmyelinated fibers $\left({ }^{* * * *} p<0.001,{ }^{* * *} p<0.005,{ }^{*} p<0.05\right.$, vehicle vs bumetanide $200 \mu \mathrm{M}$ ). Scale bar, $500 \mu \mathrm{m}$. Data are presented as the mean \pm SEM.

bumetanide-administered rats compared with those that received vehicle ( $p<0.01$; Fig. $6 A-C)$. Our results also demonstrate that the percentage of FG+ DRG neurons that were colabeled with NF200+ were decreased in bumetanide-treated animals $(p<0.001)$, corroborating that axonal regeneration of $\mathrm{NF} 200+$ myelinated neurons was prevented or delayed due to NKCC1 blockade (Fig. 6D-F). Morphometric analysis of FG+labeled neurons also confirmed the differential effect of bumetanide on DRG neuron subpopulations. We observed a decrease in the proportion of large FG + neurons, as evidenced by a shift to the left in the size frequency distribution (Fig. $6 \mathrm{~J}$ ).

As Parv-expressing neurons innervate the muscle spindles, they are considered to be muscular proprioceptors in the DRG (Celio, 1990). Thereby, to further discriminate the effect of NKCC1 blockade on subpopulations of myelinated fibers, we analyzed FG + neurons colabeled for NF200+ and Parv + . We observed that there was no difference in the number of Parv+ DRG neurons between bumetanide- and vehicle-treated groups, suggesting that the bumetanide effect mainly affects NF200+/ Parv- neurons. Indeed, the regeneration of Parv+ proprioceptive neurons was poor at the short interval assessed independently of treatment (vehicle or bumetanide; Fig. 6G-I).

To elucidate whether the bumetanide effect is specific for sensory neurons, we also assessed the regeneration of motor axons by looking at FG-retrotraced spinal motoneurons at 16 DPI after sciatic nerve cut and suture. The results showed no significant difference in the total number of FG + motoneurons between vehicle- and bumetanide-administered groups. In conclusion, the present results show that increased NKCC1 activity after peripheral nerve injury is a relevant factor for the regeneration of a selective population of myelinated sensory fibers, not including the proprioceptive ones.

\section{Bumetanide administration delays recovery of compound nerve action potentials}

Nerve conduction tests performed at 3 and 7 DPI showed complete denervation of the injured hindlimb (Fig. 7A). CNAPs were first recorded at $14 \mathrm{DPI}$ in vehicle-injected rats with a mean amplitude of $\sim 5 \%$ of control values. Bumetanide-injected rats showed significantly lower CNAP amplitudes from 14 to $28 \mathrm{DPI}$, demonstrating that bumetanide treatment slowed the regeneration of large myelinated fibers that contribute to the CNAP. After 2 months, CNAP amplitude in bumetanide rats was similar to that of vehicle-treated rats.

\section{Bumetanide administration delays sensory reinnervation after nerve injury}

After sciatic nerve section and suture repair, the early recovery of sensory responses in the hindpaw is mediated by collateral sprouts of the intact saphenous nerve fibers, which rapidly reinnervate the sciatic denervated skin from medial to central areas of the hindpaw, whereas later regeneration of sciatic nerve fibers allows the recovery of responses at central and lateral areas (Cobianchi et al., 2013, 2014). Pinprick testing was performed to assess the functional extension of collateral sprouting (medial stimulated areas) and sciatic nerve reinnervation (lateral stimulated areas) in bumetanide-treated rats. Positive responses to pinprick stimulation at medial areas similarly increased in bumetanide- and vehicle-injected rats from 3 to $21 \mathrm{~d}$ after sciatic nerve section (Fig. $7 B$, left), showing that the inhibition of 

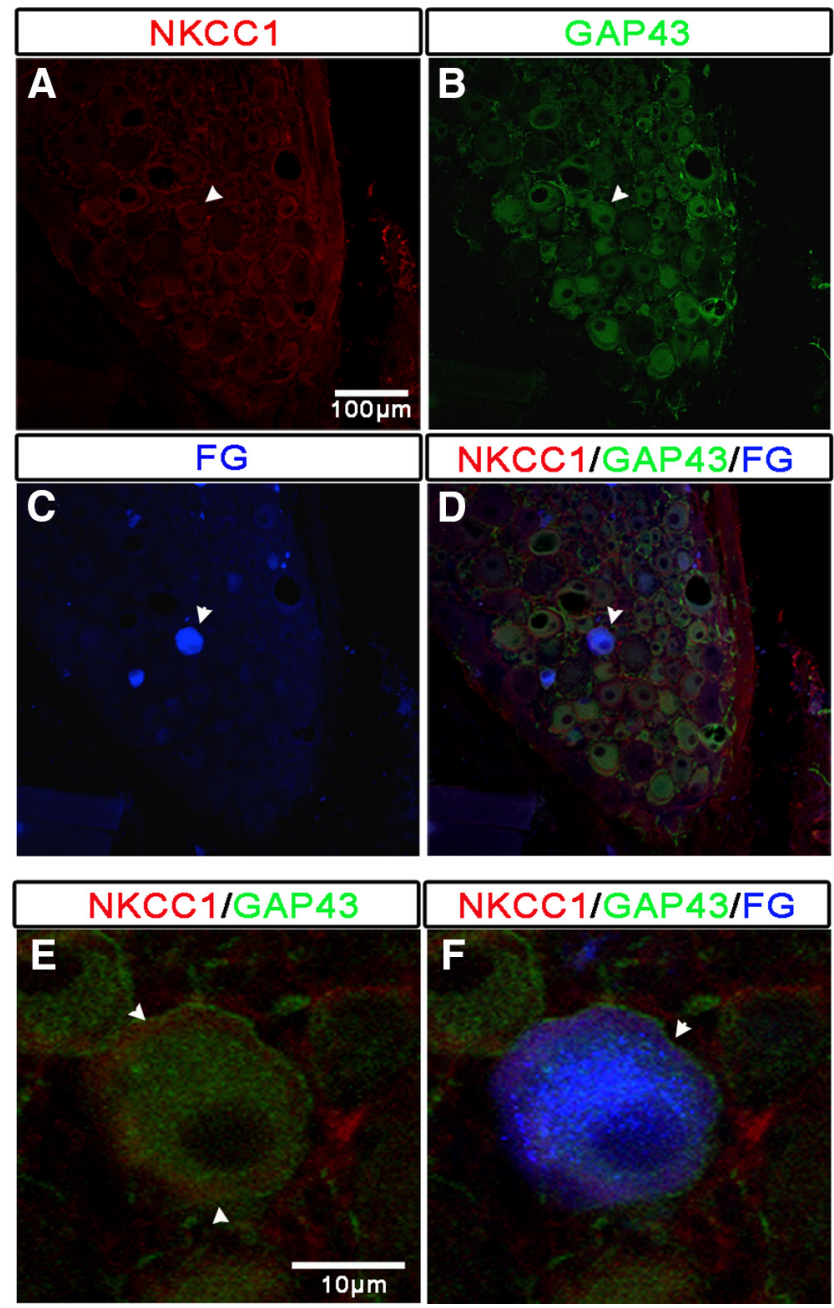

Figure 5. FG + neurons coexpress GAP43 and NKCC1. A-C, Confocal images of a representative DRG of a vehicle-treated animals at $16 \mathrm{~d}$ after nerve injury: NKCC1 expression in neuron membranes $(\boldsymbol{A})$, neurons expressing GAP43 $(\boldsymbol{B})$, and FG + neurons $(\boldsymbol{C})$. D, Merged images show that FG + neurons expressed both membrane NKCC1 and GAP43. Scale bar, $100 \mu \mathrm{m} . \boldsymbol{E}, \boldsymbol{F}$, Detailed photograph of a DRG neuron (pointed by arrowhead in $\boldsymbol{A}-\boldsymbol{D}$ ) coexpressing NKCC1 and GAP43 $(\boldsymbol{E})$ and FG $(\boldsymbol{F})$. Scale bar, $10 \mu \mathrm{m}$.

NKCC1 function was not significantly affecting collateral sprouting. At these times, sciatic areas were still denervated, since recovery of responses at lateral areas started by 21 DPI in vehicle-injected rats, and then lateral areas were progressively reinnervated from proximal to distal (Fig. 7B, right). Bumetanide-treated rats showed a delay in the return of pinprick responses, indicating a delay of reinnervation by regenerating sciatic nerve axons.

Mechanical thresholds measured in the medial site of vehicle rats (Fig. $7 B$, left) rapidly decreased to less than half the contralateral values from 3 DPI, and remained low during the follow-up, reflecting a state of chronic adjacent hyperalgesia induced by collateral sprouting, as previously reported (Cobianchi et al., 2014). In contrast, rats receiving bumetanide during the first 2 weeks showed a significantly higher mechanical threshold from 3 to 21 DPI $(p<0.001$ at 3 and 7 DPI; $p<0.05$ at 14 and 21 DPI), demonstrating a reduction of saphenous nerve sensitization. In parallel, the measurement of mechanical sensory threshold in the lateral side (Fig. 7C) showed a time course from hypoesthesia to hyperalgesia reflecting progressive sciatic regeneration, similar to the pinprick test. Responsiveness to mechanical stimuli reappeared from 21 DPI in vehicle-injected rats, and from 28 DPI in bumetanide-treated rats. At later times of regeneration, withdrawal thresholds lowered to $\sim 50 \%$ of the contralateral paw, indicating hyperalgesia as previously documented after reinnervation (Cobianchi et al., 2014).

These results demonstrate that blocking NKCC1 activity markedly delays sensory nerve regeneration, since the recovery of sciatic sensory thresholds parallels the progressive reinnervation of the skin. Bumetanide administration also transiently reduced the adjacent hyperalgesia as measured at saphenous nerve territory, as previously demonstrated (Mòdol et al., 2014), however, without affecting the progression of collateral sprouting. This suggests that, even if NKCC1 function is involved in excitability and sensitization of sensory neurons after nerve injury, it is not involved in intact unmyelinated fiber sprouting.

To further corroborate the delayed sensory regeneration caused by NKCC1 blockade, we analyzed the skin innervation of the lateral footpad by immunohistochemistry. Two months after sciatic nerve section and repair, samples from vehicle-treated rats showed small nerve bundles of PGP + nerve fibers in the dermis, with some also showing bundles of NF200+ nerve fibers, that reached the subepidermal nerve plexus (Fig. 8). Intraepidermal nerve fibers were rarely observed, and those present penetrated the thin epidermis a short distance. In footpads of bumetanidetreated rats, the number of reinnervating fibers was lower, and few NF200+ fibers were observed, resulting in significantly decreased density measurement compared with control (nonlesioned) and vehicle-treated groups $(p<0.001$; Fig. $8 B)$. Very few IENFs were counted, and large areas of the epidermis remained denervated.

\section{Bumetanide administration decreases ERK1/2 and JNK1/2/3 expression in DRG neurons}

To assess the potential mechanisms by which the blockade of NKCC1 activity affects regeneration in the myelinated DRG sensory subpopulation, we quantified the changes in expression of the phosphorylated state of MAPKs, as follows: ERK1/2, p38 MAPK, and JNK1/2/3 by Western blot and ELISA (Fig. $9 A-D$ ). $\mathrm{WB}$ analyses showed that bumetanide administration in vivo mainly prevented increased expression of pJNK when compared with naive samples ( $p<0.05$, one-way ANOVA). Additionally, although increased expression of pERK1/2 is also observed $\left(+p<0.05\right.$ and $\left.{ }^{\star} p<0.05\right)$ when compared with naive groups, no statistical differences are observed when compared with bumetanide-treated animals. To further explore the changes in MAPKs, we also performed an ELISA assay. The three MAPKs analyzed showed a noticeable increase after nerve injury in control rats (Fig. 9D). In contrast, animals treated with bumetanide showed a marked decrease in the expression of both ERK1/2 and JNK1/2/3 ( $p<0.02$, two-way ANOVA) compared with animals given vehicle (one-way ANOVA: ERK1/2, $p=0.06$; JNK1/2/3, $p<0.01$; Fig. 9D).

Increased ERK signaling is a relevant feature of glial cell response to axonal damage (Harrisingh et al., 2004). Thereby, to assess whether the decreased pERK1/2 expression in bumetanideadministered animals could be mediated by peripheral glial cells (Schwann cells and satellite cells), we added cytosine arabinoside (AraC) to the culture medium to inhibit their proliferation and to isolate the effects of NKCC1 blockade on MAPK expression in DRG neurons (Liu et al., 1986). Our results show that only the decrease in $\mathrm{pJNK} 1 / 2 / 3$ expression is affected by bumetanide (bumetanide $50 \mathrm{~mm}$ and AraC) administration compared with the vehicle (DMSO and AraC)-treated cultures $(p<0.05)$, while pERK1/2 expression remains unchanged (Fig. $9 E$ ). This last find- 

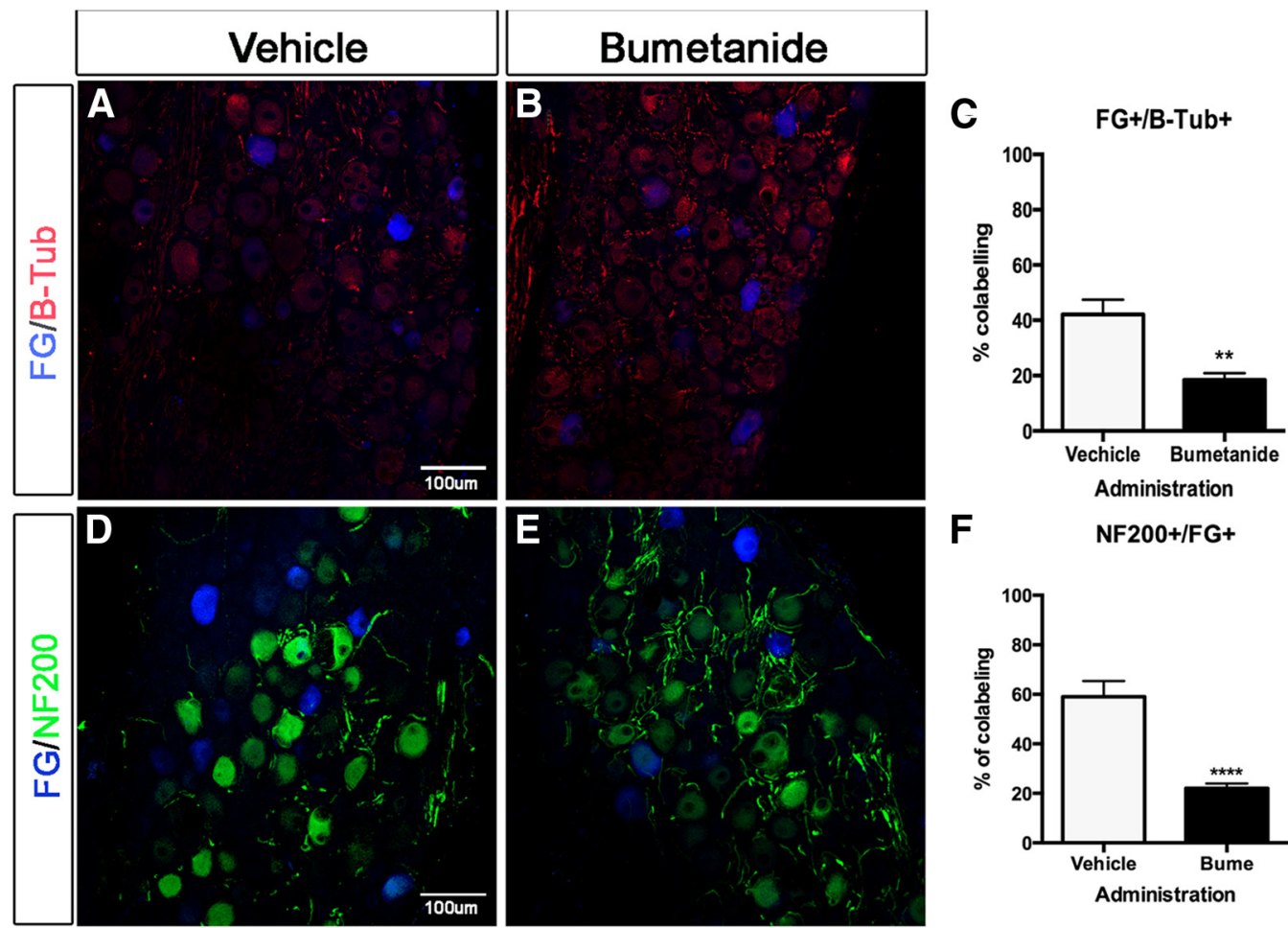

$\mathbf{F}$

NF200+/FG+
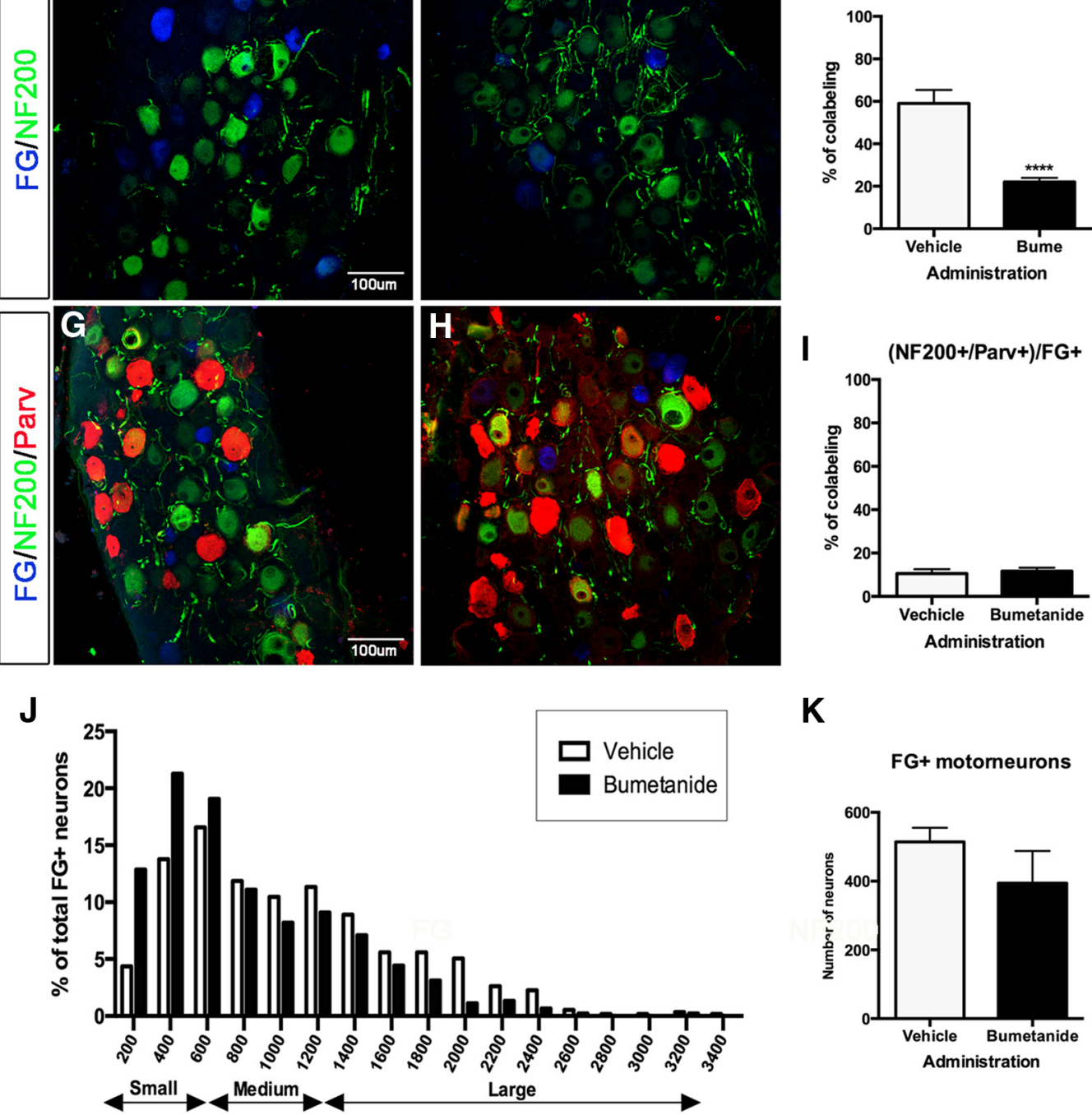

K

FG+ motorneurons

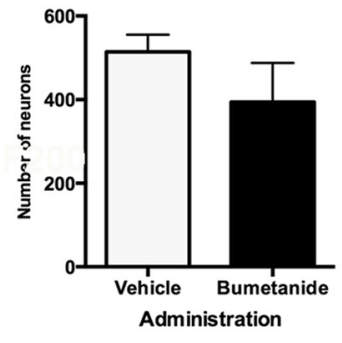

Cross Sectional Area $\left(\mu \mathrm{m}^{2}\right)$

Figure 6. Bumetanide administration decreases myelinated fiber regeneration in vivo. Colabeling of $F G+D R G$ neurons comparing unmyelinated to myelinated fiber regeneration under bumetanide administration. $\boldsymbol{A}, \boldsymbol{B}, \boldsymbol{D}, \boldsymbol{E}, \boldsymbol{G}, \boldsymbol{H}$, Confocal images of FG $+/ \boldsymbol{\beta}$-Tub $+(\boldsymbol{A}, \boldsymbol{B}), \mathrm{FG}+/ \mathrm{NF} 200+(\boldsymbol{D}, \boldsymbol{E})$, and FG $+/ \mathrm{NF} 200+/$ Parv $+(\boldsymbol{G}, \boldsymbol{H})$ DRG neurons in vehicle- or bumetanide-treated animals after injury. Scale bar, $100 \mu \mathrm{m}$. $C$, Colabeling with $\beta$-Tub showed a decrease in the number of regenerating neurons that are $\mathrm{FG}+\left({ }^{* *} p<0.01\right)$ in animals to which bumetanide had been administered. $\boldsymbol{F}$, Interestingly, an important decrease of regenerating neurons ( $\mathrm{FG}+$ ) was found for NF200+ neurons in bumetanide-treated animals (**** $p<0.001)$. $I$, Quantification of the percentage of $\mathrm{FG}+$ and NF200 + neurons colabeled with Parv. Bumetanide did not affect the regeneration of Parv + proprioceptive neurons. J, Size distribution histogram of FG + DRG neurons after sciatic nerve injury. Neurons in fivesections from four rats for each condition (vehicleand bumetanide) were counted for the analysis. A shiftt the leftis observed in bumetanide-administered animals. $\boldsymbol{K}$, Bumetanide administration did not affect motoneuron regeneration. Data are presented as the mean \pm SEM. 
A

\section{L5 L4 L3}
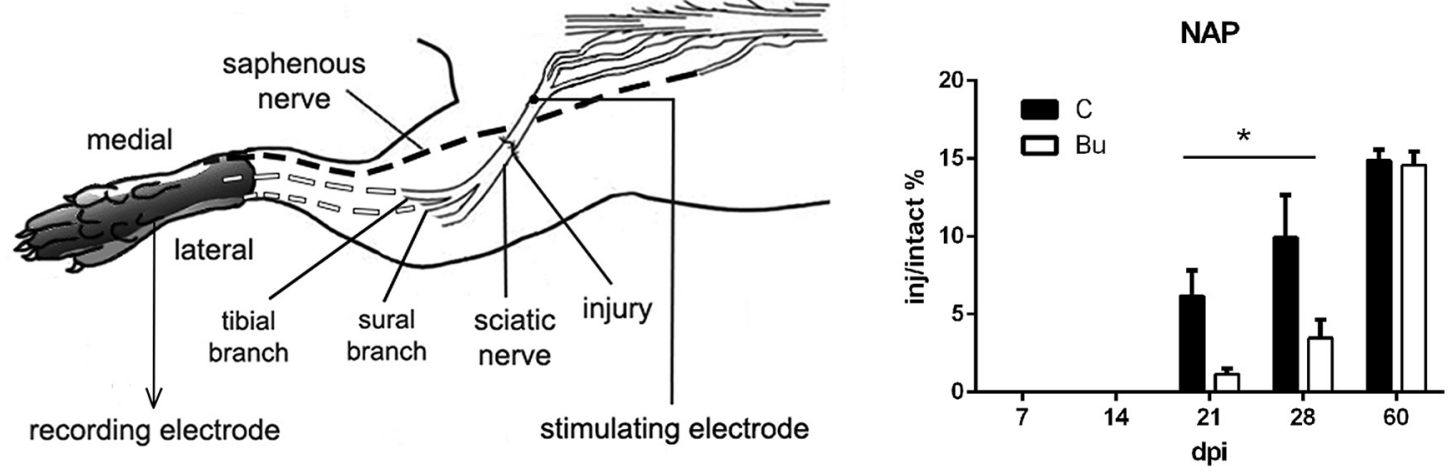

B

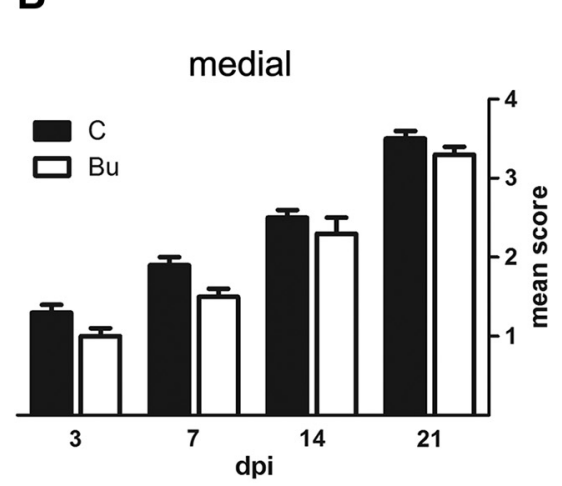

PINPRICK TEST
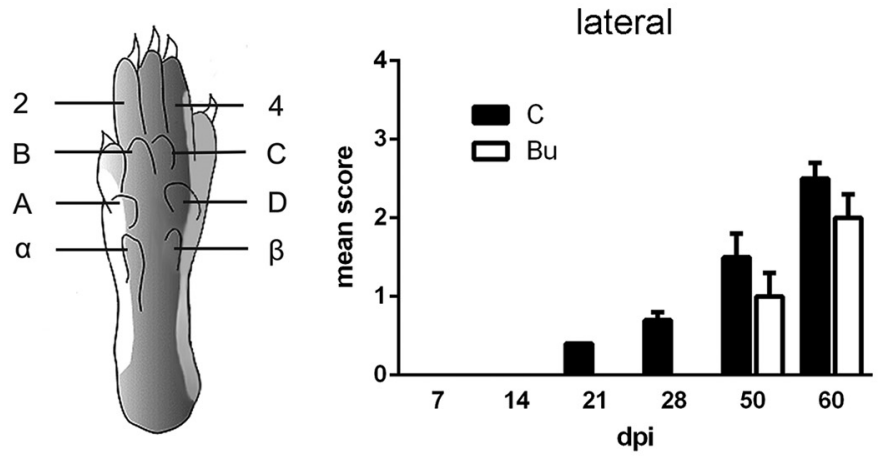

C

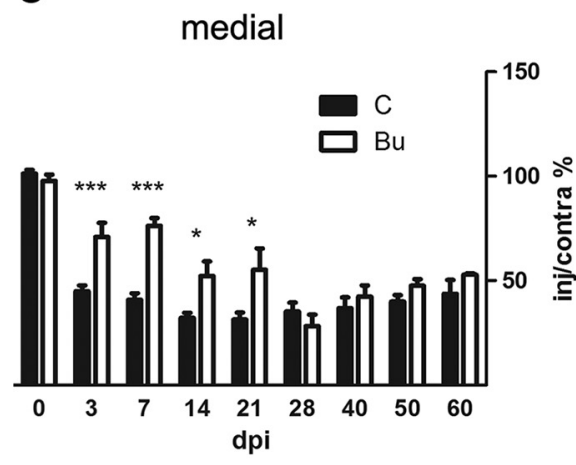

VON FREY TEST
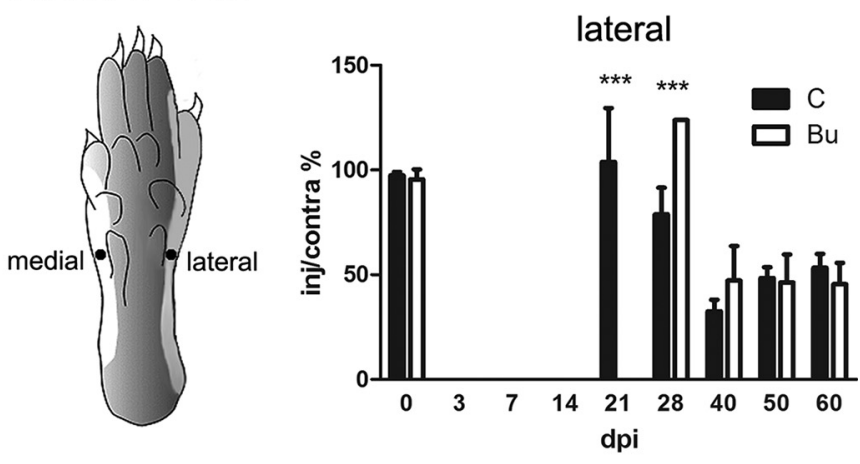

Figure 7. Bumetanide administration delays the return of sensory responses after sciatic nerve lesion. $A$, The mean amplitude of the CNAPs recorded in the lateral plantar nerve was lower in bumetanide-treated $(\mathrm{Bu})$ rats that in control $(\mathrm{C})$ rats at 21 and $28 \mathrm{~d}$ after nerve section and repair. $\boldsymbol{B}$, Collateral sprouting (left) was not significantly affected by bumetanide administration as the responses of Bu rats to pinprick indicated a similar progression of early nociceptive reinnervation compared with $C$ rats in the four medial plantar areas ( $\alpha, A, B$ footpads and toe 2). On the contrary, nociceptive sciatic nerve regeneration (right) was delayed as lateral areas ( $\beta, D, C$ footpads and toe 4 ) were reinnervated later in Bu rats compared with C rats. C, Changes in mechanical sensory thresholds in the saphenous medial (left) and sciatic lateral (right) test sites of the paw, assessed after sciatic nerve injury. The early mechanical hyperalgesia (left) was transiently reduced by bumetanide treatment, which was indicated by significantly higher thresholds of Bu rats (*** $p<0.001$ at 3 and 7 DPI; * $p<$ 0.05 at 14 and $21 \mathrm{DPI}$ ). Lateral responses to mechanical stimuli (right) reappeared at $21 \mathrm{DPI}$ in vehicle $(\boldsymbol{C})$ but not in bumetanide-injected rats, while only a few Bu rats (20\%) recovered responses at $28 \mathrm{DPI}$ compared with C rats (75\%).

ing suggests that the changes in pERK1/2 expression observed in vivo might be mediated by glial cells (Schwann cells or satellite cells) rather than neurons. To corroborate this result in vivo, we also performed an immunohistochemical analysis in adult animals to which bumetanide $(30 \mathrm{mg} / \mathrm{kg})$ or vehicle had been administered, by assessing the colabeling of GFAP (a Schwann cell marker) and pERK1/2 or NF200+ cells and pJNK in DRGs (Fig. $9 F, G)$. The fact that pERK1/2 signaling is colocalized with GFAP further suggests that changes in pERK1/2 expression are mediated by glial cells and that the effects of bumetanide administration on neurons are dependent upon JNK signaling.

\section{Discussion}

Our results demonstrate that increased pNKCC1 after axotomy is important for DRG neuron regeneration, particularly for myelinated fibers, without affecting nonmyelinated neurons. Our results in vitro point out that blocking NKCC1 activity by bumetanide or furosemide administration to DRG cultures decreases the neurite outgrowth of NF200+ neurons. In vivo, we observed a decrease in the percentage of regenerating NF200+ DRG sensory neurons together with a decrease in the proportion of large neurons retrolabeled with FG, as evi- 
A
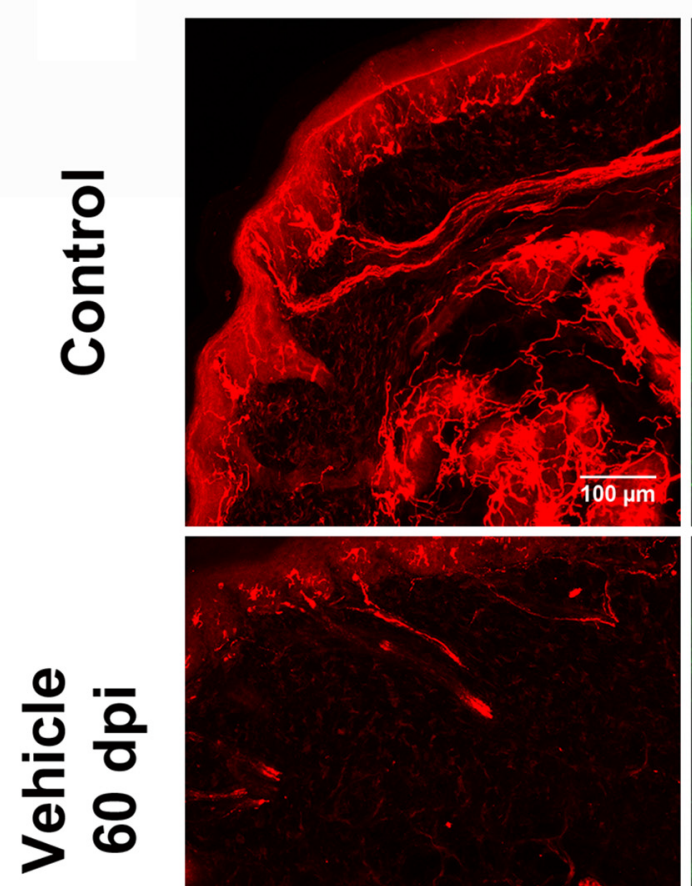

毫䇏

B
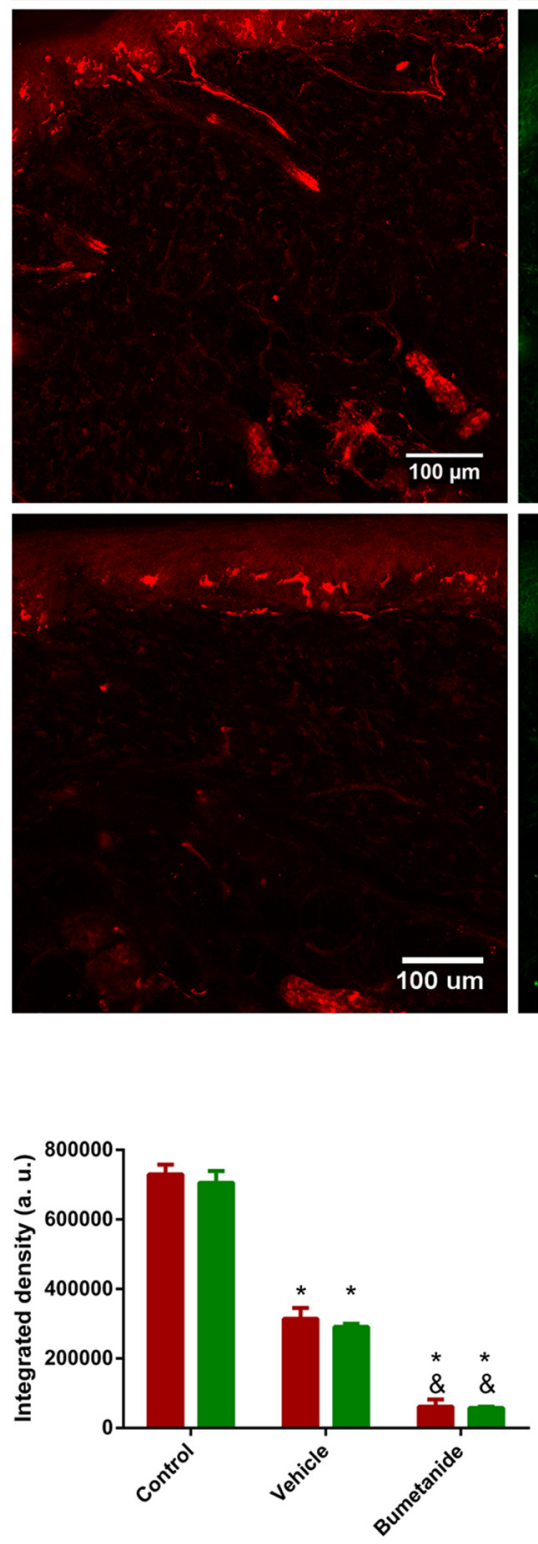

NF200
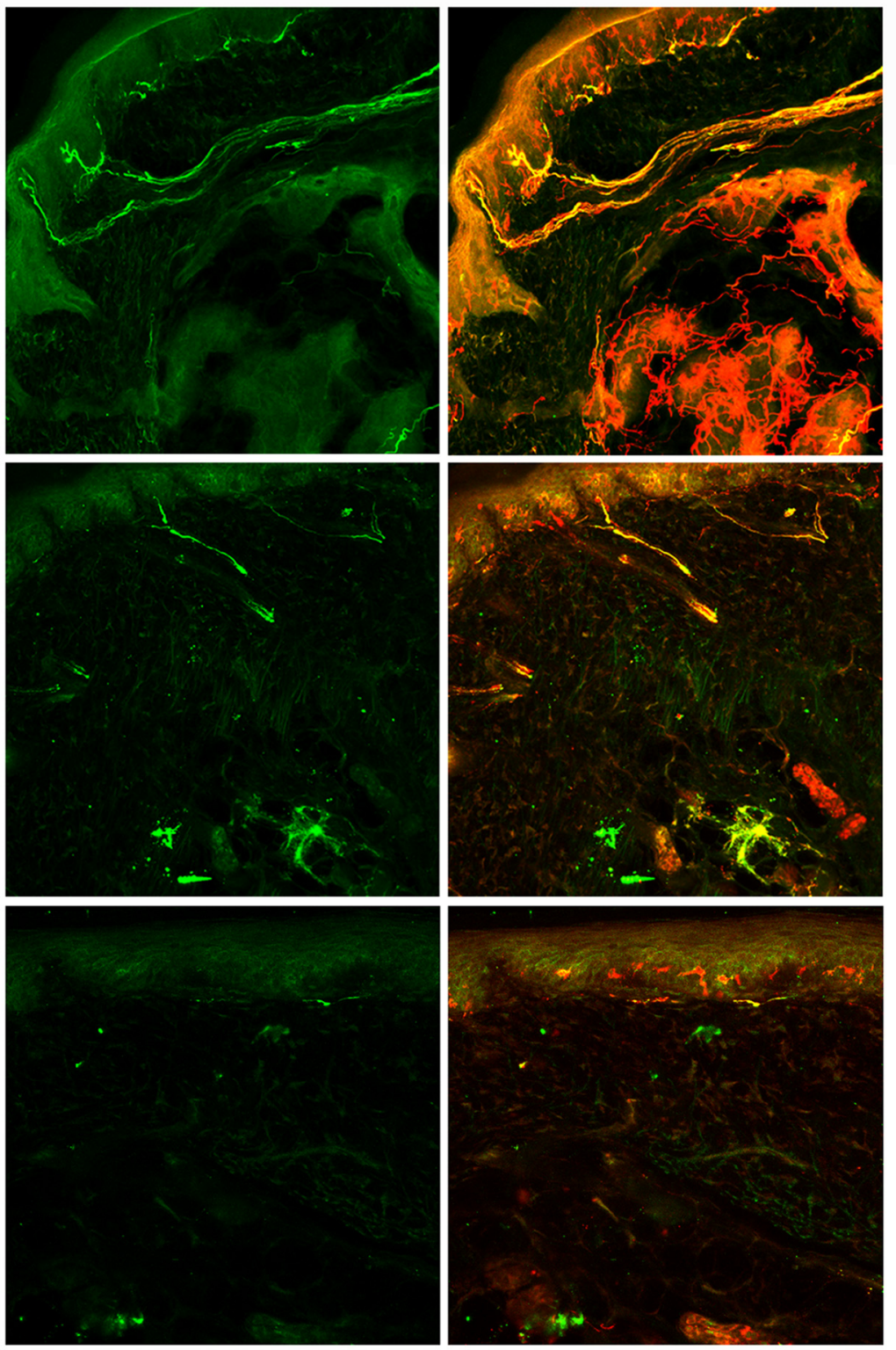

C

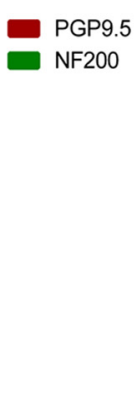

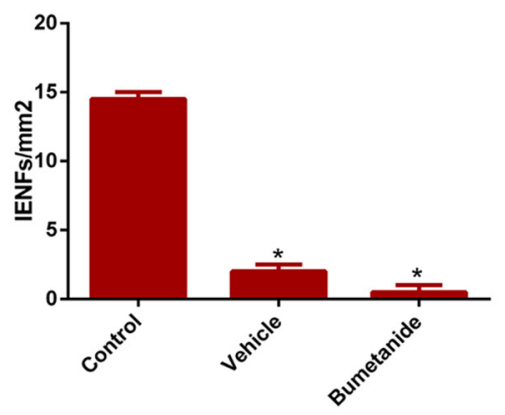

Figure 8. A, Confocal micrographs of footpad sections immunolabeled against PGP9.5 and NF200 in samples of intact controls, and vehicle- and bumetanide-treated rats at 60 DPI. The density of regenerated nerve profiles in the subepidermal plexus was more abundant in vehicle-treated rats than in bumetanide-treated rats. The presence of NF200+ profiles was also lower in bumetanide-treated rats. There were a few small fibers entering the epidermis, where an increased number of Langerhans cells, positive for PGP9.5, was observed at 2 months after sciatic nerve injury. $\boldsymbol{B}$, Measurement of the integrated density of immunolabeling for PGP9.5 and NF200 in the footpad. Significantly lower values were found in bumetanide-treated rats compared with the vehicle-treated rats ( ${ }^{*} p<0.001$ vs control; \& $<0.001$ vs vehicle). C, Counts of IENFs labeled for PGP9.5 ( ${ }^{*} p<0.001$ vs control). Scale bar, $100 \mu \mathrm{m}$. Data are presented as the mean \pm SEM. 
A

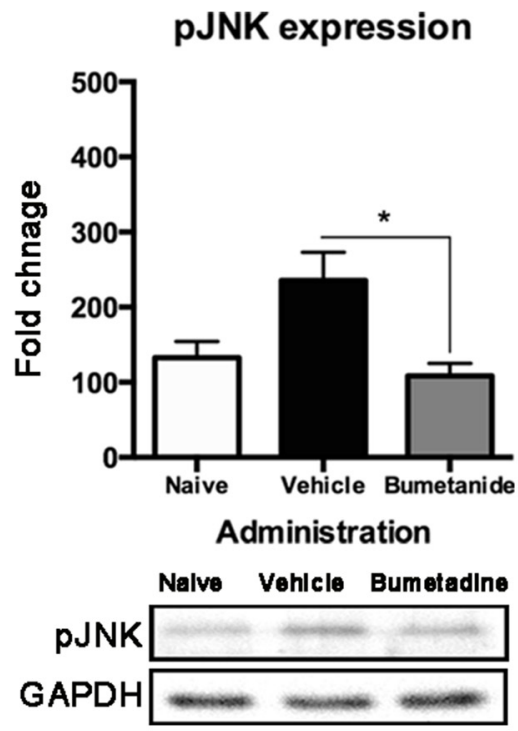

B

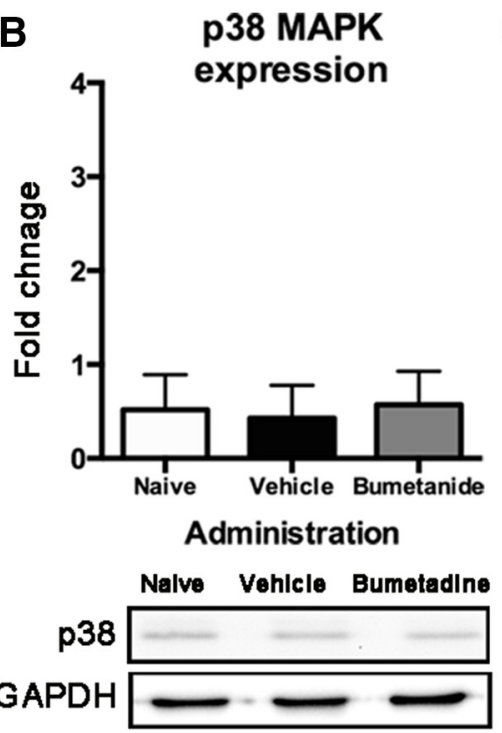

C

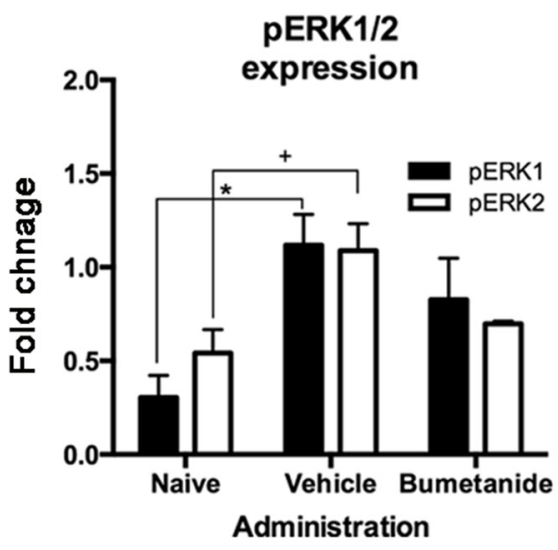

Naive Vehicle Bumetadine

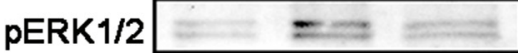

GAPDH
ELISA

D

${ }^{100} 7$
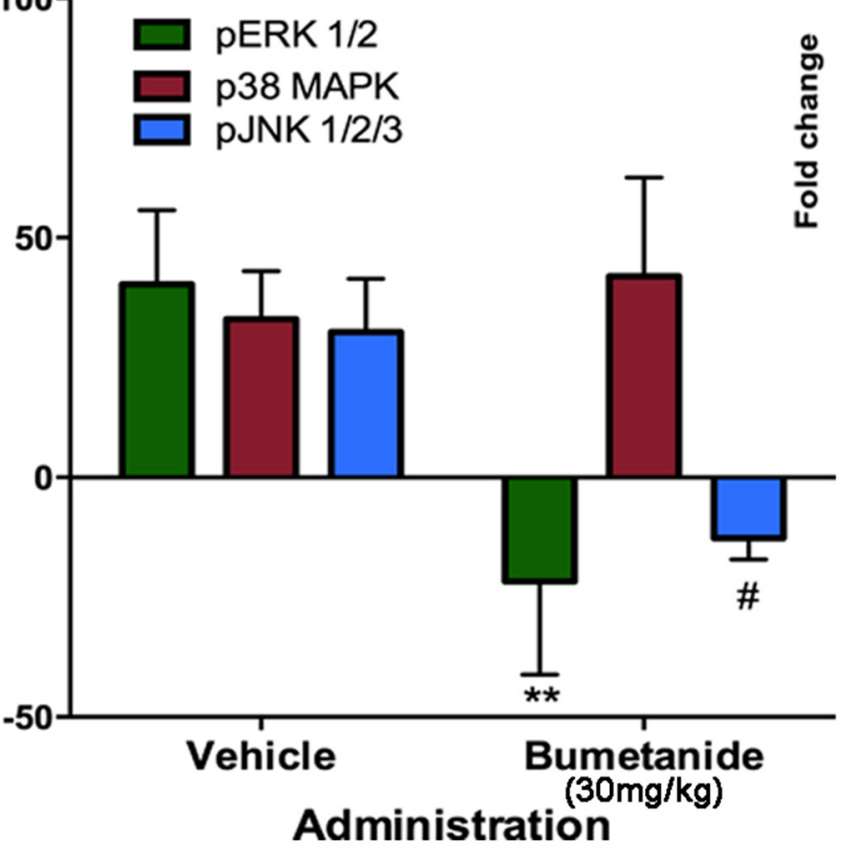

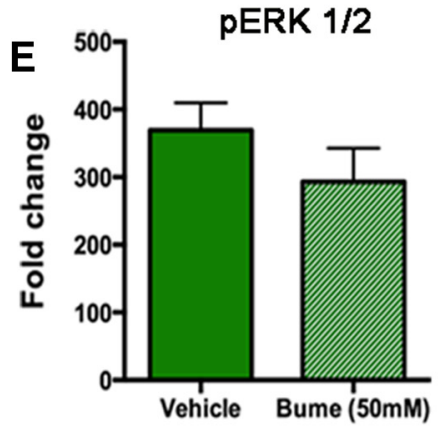

Administration

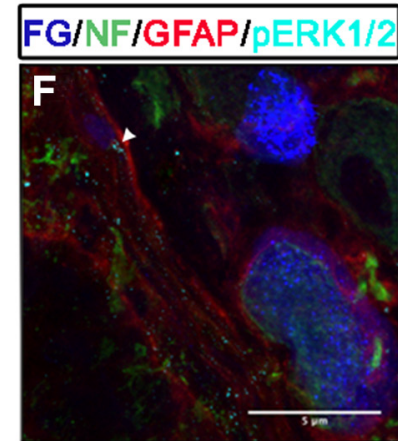

pJNK 1/2/3

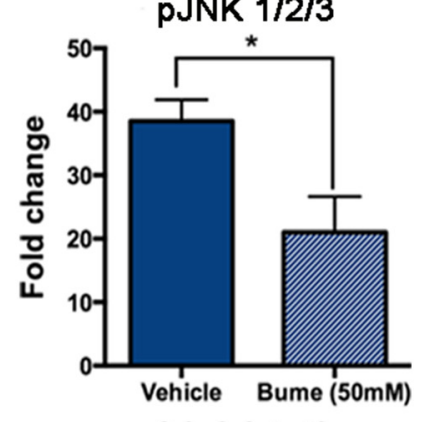

Administration

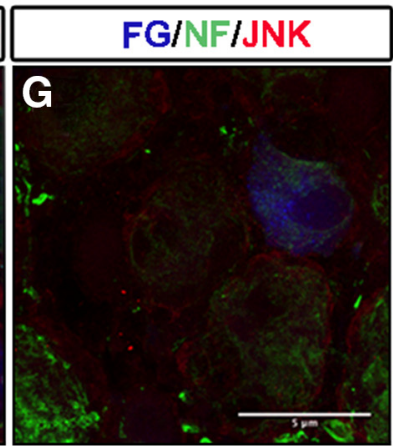

Figure 9. Bumetanide (Bume) administration decreases the expression of pJNK1/2/3 in vitro and in vivo. Changes in the expression of different MAPKs assessed by Western blot and ELISA. A-C, Representative blots and quantification of $\mathrm{pJNK} 1 / 2 / 3, \mathrm{p} 38 \mathrm{MAPK}$, and pERK1/2 expression. Bumetanide administration prevented the increase of $\mathrm{pJNK} 1 / 2 / 3$ observed in injured animals $\left({ }^{*} p<0.05\right.$, one-way ANOVA). Increased expression of pERK1/2 is also observed in injured animals compared with naive animals ( ${ }^{*} p<0.05,+p<0.05$, one-way ANOVA). $\boldsymbol{D}$, ELISA results showed increased expression of $p E R K 1 / 2, p 38$, and pJNK1/2/3 in DRGs after nerve injury in vehicle-treated animals. Bumetanide administration reduced the expression of pERK1/2(\#p $=0.06)$ and $p J N K 1 / 2 / 3\left({ }^{* *} p<\right.$ 0.01) without affecting that of p38MAPK. $E$, DRG-dissociated cultures with the cell cycle inhibitor AraC were performed to isolate the effect of bumetanide administration on MAPK expression in neurons. Bumetanide administration decreased pJNK1/2/3 expression $\left({ }^{*} p<0.05\right)$, but not $p E R K 1 / 2$ expression. $F, G$, Immunohistochemical localization of $p E R K 1 / 2$ also confirmed its expression in satellite cells surrounding DRG neurons $(\boldsymbol{F})$, while JNK was expressed mainly in DRG neuron somas $(\boldsymbol{G})$.

denced by a shift to the left in the size frequency distribution. Interestingly, bumetanide-affected myelinated fibers were not parvalbumin positive, suggesting that myelinated fibers affected by NKCC1 blockade are not proprioceptive. The functional in vivo study showed that bumetanide treatment delayed the reappearance of sciatic responses due to reinnervation by regenerating axons, but did not affect collateral sprouting, although it reduced hyperalgesia after injury. The delay in functional reinnervation was associated with lower skin reinnervation. We also found that the inhibitory action of NKCC1 blockade on axonal regeneration was related to the involvement of JNK pathway signaling in DRG neurons.

Blockade of NKCC1 activity impairs regeneration of sensory myelinated fibers

NKCC1 is a cation-chloride cotransporter that is particularly relevant in setting the $\mathrm{Cl}^{-}$equilibrium potential in early stages of 
development when GABA is excitatory and neurons are still migrating (Ben-Ari et al., 2007). Under physiological conditions, the sensory neuron reversal potential of $\mathrm{Cl}^{-}$is approximately $-40 \mathrm{mV}$ (Sung et al., 2000). Following nerve injury, it turns to $-20 \mathrm{mV}$ due to the increased expression of pNKCC1, resulting in a $\mathrm{Cl}^{-}$reversal potential shifted toward depolarized potentials and increased $\left[\mathrm{Cl}^{-}\right]_{\mathrm{i}}$ (Pieraut et al., 2007). In agreement with the present results, increased membrane expression of pNKCC1 after injury has been linked to the rate of regenerative growth in sensory neurons and associated to changes in $\mathrm{Cl}^{-}$accumulation (Pieraut et al., 2007). However, it is the first time that NKCC1 effects are described to selectively affect a subpopulation of large sensory neurons. Interestingly, it was reported that increased IL-6, which triggers the increase in pNKCC1 after injury, is also observed in myelinated cells (NF200+ neurons; Pieraut et al., 2011). Other studies (Liu et al., 2000, 2002; Ming et al., 2001; André et al., 2003) reported that medium- to large-diameter neurons develop an increased excitability in vivo and in vitro, and abnormal electrical activity related to axonal growth. In this context, the role of ions, such as $\mathrm{Na}^{+}$or $\mathrm{Cl}^{-}$, in the modulation of calcium signaling that participates in regeneration is generating increasing interest (Wolf et al., 2001; André et al., 2003; Waxman and Zamponi, 2014). For instance, changes in neurite growth by shifts in $\mathrm{Cl}^{-}$membrane potential have already been reported (André et al., 2003; Nishiyama et al., 2008).

Inhibition of NKCC1 activity by bumetanide administration induced a delay in sensory recovery after nerve lesion. We observed a delay in the reappearance of CNAPs and responses to mechanical stimulation in the sciatic skin territory. It is therefore plausible that bumetanide treatment mainly compromises the regrowth of myelinated injured neurons, possibly affecting $A \delta$ myelinated fibers that mediate acute pain (Basbaum et al., 2009). The decrease of action potentials in bumetanide-treated rats also supports that $\mathrm{NKCC} 1$ function is involved in sensory myelinated fiber regeneration, since the CNAP amplitude is dependent on the number of large $(A \alpha \beta)$ myelinated fibers in the nerve (Fugleholm et al., 2000; Navarro and Udina, 2009). Despite the fact that nerve fibers regenerate at random after nerve transection and repair, the sensory fibers represent approximately three-quarters of the myelinated fibers in the rat sciatic nerve, and therefore have much more contribution to the CNAP amplitude than motor fibers. In contrast, the growth of collateral saphenous sprouts was not significantly affected by bumetanide treatment. In fact, bumetanide also transiently improved the mechanical hyperalgesia related to collateral sprouting of the saphenous nerve, confirming that blocking NKCC1 activity after nerve lesion reduces the early hyperalgesic responses. Further, the modulation of A $\delta$ myelinated afferents that project to the lamina I of the spinal cord could also explain the prevention of central disinhibition via KCC2 dephosphorylation in spinal cord and higher pain projection areas (Mòdol et al., 2014).

Retrograde labeling of regenerated axons allowed quantification of a decrease in the proportion of large neurons, identified as myelinated by colabeling with NF200, that regenerated in the distal sciatic nerve of bumetanide-treated rats. Conventional histological study of epon-embedded distal nerves, harvested at the time of retrotracer application (i.e., $10 \mathrm{~d}$ postlesion), showed numerous unmyelinated and a few myelinated regenerated profiles, as expected, without differences between bumetanide-treated and untreated rats (data not shown). However, the nonspecific information provided by the histological readout precludes the identification of subpopulations of axons. Immunohistochemical analyses showed that NKCC1 is expressed in all DRG subpopu- lations before and after nerve injury. It is thus surprising that NKCC1 inhibition affects only myelinated neuron regeneration, excluding those that mediate proprioceptive information. This suggests that medium- to large-diameter DRG neurons (myelinated) must be more sensitive to changes in $\left[\mathrm{Cl}^{-}\right]$and are dependent upon $\mathrm{Cl}^{-}$fluctuations to regenerate, while the ability of small-diameter neurons (nonmyelinated) to regrow may rely on other mechanisms.

\section{Bumetanide blockade of NKCC1 activity decreases the expression of $\mathrm{pJNK} 1 / 2 / 3$}

MAPKs are relevant signaling molecules that respond to extracellular stimuli and generate differential effects in the cell response. In DRG neurons, MAPKs have been implicated in axonal regeneration mechanisms, such as the following: retrograde signaling (Rishal and Fainzilber, 2014); glial cell dedifferentiation (Napoli et al., 2012); or soma gene transduction (Waetzig et al., 2006; Raivich and Makwana, 2007). Although increased IL-6 levels after axotomy have been postulated as a relevant factor that triggers increased pNKCC1 expression (Pieraut et al., 2011), the downstream mechanisms that mediate NKCC1 effects have not been yet explored. Our results demonstrate that bumetanide administration decreased both pERK1/2 and pJNK1/2/3 expression in DRGs without modifying p38 MAPK expression. In this sense, previous studies (Obata et al., 2004) showed increased p38MAPK expression in small-diameter sensory fibers after injury. Thus, the lack of effects in p38 MAPK expression may coincide with the fact that we did not observe changes in neurite outgrowth and regeneration of unmyelinated cells (small diameter) treated with bumetanide.

We found a decrease in $\mathrm{pJNK} 1 / 2 / 3$ expression in vitro and in vivo under NKCC1 blockade with bumetanide. The role of JNK kinases in regeneration has already been highlighted in in vitro studies (Waetzig et al., 2006). For instance, increased pJNK levels in DRG somas after injury is important for axonal growth of sensory neurons (Kenney and Kocsis, 1998; Lindwall et al., 2004). However, it is the first time that changes in NKCC1 activity in DRG neurons have been shown to modulate the expression of MAPKs after injury.

On the other hand, activation of the MERK/ERK/Raf kinase pathway has been linked to peripheral glial cell differentiation after injury (Harrisingh et al., 2004; Napoli et al., 2012). In our study, immunohistochemical localization of pERK also confirmed its expression in satellite cells surrounding the DRG neurons. Furthermore, the administration of the cell cycle inhibitor AraC in vitro affected only pJNK $1 / 2 / 3$ expression, but not pERK $1 / 2$ expression, in cultures in which bumetanide $(50 \mu \mathrm{M})$ had been administered. Therefore, it is plausible that the downregulation of pERK1/2 expression observed in animals treated with bumetanide could be triggered by satellite or Schwann cells (Harrisingh et al., 2004). However, the fact that bumetanide administration in vivo also induced changes in pERK1/2 expression suggests that this effect might be mediated by the modulation of neuron-glial communication and triggered by changes in electrical activity induced by the modulation of NKCC1, which is not expressed in glial cells.

Together, the present results reinforce the relevance of NKCC1 activity in the regeneration of myelinated cells by modifying the JNK activity, further suggesting that NKCC1 modulation of $\mathrm{Cl}^{-}$ membrane potential is a key factor in the modulation of MAPK signaling. 


\section{References}

Abe N, Cavalli V (2008) Nerve injury signaling. Curr Opin Neurobiol 18: 276-283. CrossRef Medline

Allodi I, Guzmán-Lenis MS, Hernàndez J, Navarro X, Udina E (2011) In vitro comparison of motor and sensory neuron outgrowth in a $3 \mathrm{D}$ collagen matrix. J Neurosci Methods 198:53-61. CrossRef Medline

André S, Boukhaddaoui H, Campo B, Al-Jumaily M, Mayeux V, Greuet D, Valmier J, Scamps F (2003) Axotomy-induced expression of calciumactivated chloride current in subpopulations of mouse dorsal root ganglion neurons. J Neurophysiol 90:3764-3773. CrossRef Medline

Basbaum AI, Bautista DM, Scherrer G, Julius D (2009) Celullar and molecular mechanisms of pain. Cell 139:267-284. CrossRef Medline

Ben-Ari Y, Gaiarsa JL, Tyzio R, Khazipov R (2007) GABA: a pioneer transmitter that excites immature neurons and generates primitive oscillations. Physiol Rev 87:1215-1284. CrossRef Medline

Ben-Ari Y, Woodin MA, Sernagor E, Cancedda L, Vinay L Rivera C, Legendre P, Luhmann HJ, Bordey A, Wenner P, Fukuda A, van den Pol AN, Gaisar JL, Cherubini E (2012) Refuting the challenges of the developmental shift of polarity of GABA actions: GABA more exciting tan ever! Front Cell Neurosci 6:35. CrossRef Medline

Bradke F, Fawcett JW, Spira ME (2012) Assembly of a new growth cone after axotomy: the precursor to axon regeneration. Nat Rev Neurosci 13:183193. CrossRef Medline

Celio MR (1990) Calbindin D-28k and parvalbumin in the rat nervous system. Neuroscience 35:375-475. CrossRef Medline

Cervero F, Laird JM, García-Nicas E (2003) Secondary hyperalgesia and presynaptic inhibition: an update. Eur J Pain 7:345-351. CrossRef Medline

Cobianchi S, Casals-Diaz L, Jaramillo J, Navarro X (2013) Differential effects of activity dependent treatments on axonal regeneration and neuropathic pain after peripheral nerve injury. Exp Neurol 240:157-167. CrossRef Medline

Cobianchi S, de Cruz J, Navarro X (2014) Assessment of sensory thresholds and nociceptive fiber growth after sciatic nerve injury reveal the differential contribution of collateral reinnervation and nerve regeneration to neuropathic pain. Exp Neurol 255:1-11. CrossRef Medline

Fugleholm K, Schmalbruch H, Krarup C (2000) Post reinnervation maturation of myelinated nerve fibers in the cat tibial nerve: chronic electrophysiological and morphometric studies. J Peripher Nerv Syst 5:82-95. CrossRef Medline

Gundersen HJ (1986) Stereology of arbitrary particles. A review of unbiased number and size estimators and the presentation of some new ones, in memory of William R. Thompson. J Microsc 143:3-45. Medline

Harrisingh MC, Perez-Nadales E, Parkinson DB, Malcolm DS, Mudge AW, Lloyd AC (2004) The Ras/Raf/ERK signalling pathway drives Schwann cell dedifferentiation. EMBO J 23:3061-3071. CrossRef Medline

Kenney AM, Kocsis JD (1998) Peripheral axotomy induces long-term c-Jun amino-terminal kinase-1 activation and activator protein-1 binding activity by c-Jun and junD in adult rat dorsal root ganglia in vivo. J Neurosci 18:1318-1328. Medline

Lindwall C, Kanje M (2005) Retrograde axonal transport of JNK signaling molecules influence injury induced nuclear changes in p-Jun and ATF3 in adult rat sensory neurons. Mol Cell Neurosci 29:269-282. CrossRef Medline

Lindwall C, Dahlin L, Lundborg G, Kanje M (2004) Inhibition of c-Jun phosphorylation reduces axonal outgrowth of adult rat nodose ganglia and dorsal root ganglia sensory neurons. Mol Cell Neurosci 27:267-279. CrossRef Medline

Liu CN, Wall PD, Ben-Dor E, Michaelis M, Amir R, Devor M (2000) Tactile allodynia in the absence of C-fiber activation: altered firing properties of DRG neurons following spinal nerve injury. Pain 85:503-521. CrossRef Medline

Liu CN, Devor M, Waxman SG, Kocsis JD (2002) Subthreshold oscillations induced by spinal nerve injury in dissociated muscle and cutaneous afferents of mouse DRG. J Neurophysiol 87:2009-2017. Medline

Liu L, Halfter W, Layer PG (1986) Inhibition of cell proliferation by cytosinearabinoside and its interference with spatial and temporal differentiation patters in the chick retina. Cell Tissue Res 244:501-513. Medline

Mandolesi G, Madeddu F, Bozzi Y, Maffei L, Ratto GM (2004) Acute physiological response of mammalian central neurons to axotomy: ionic regulation and electrical activity. FASEB J 18:1934-1936. CrossRef Medline

Michael GJ, Averill S, Nitkunan A, Rattray M, Bennett DL, Yan Q, Priestley JV (1997) Nerve growth factor treatment increases brain-derived neurotrophic factor selectively in TrkA expressing dorsal root ganglion cells and in their central terminations within the spinal cord. J Neurosci 17: 8476-8490. Medline
Ming G, Henley J, Tessier-Lavigne M, Song H, Poo M (2001) Electrical activity modulates growth cone guidance by diffusible factors. Neuron 29: 441-452. CrossRef Medline

Mòdol L, Cobianchi S, Navarro X (2014) Prevention of NKCC1 phosphorylation avoids down-regulation of KCC2 in central sensory pathways and reduces neuropathic pain after peripheral nerve injury. Pain 155:15771590. CrossRef Medline

Napoli I, Noon LA, Ribeiro S, Kerai AP, Parrinello S, Rosenberg LH, Collins MJ, Harrisingh MC, White IJ, Woodhoo A, Lloyd AC (2012) A central role for the ERK-signaling pathway in controlling Schwann cell plasticity and peripheral nerve regeneration in vivo. Neuron 73:729-742. CrossRef Medline

Navarro X, Udina E (2009) Methods and protocols in peripheral nerve regeneration ex- perimental research: part III-electrophysiological evaluation. Int Rev Neurobiol 87:105-126. CrossRef Medline

Navarro X, Verdú E, Butí M (1994) Comparison of regenerative and reinervating capabilities of diferent functional types of nerve fibers. Exp Neurol 129:217-224. CrossRef Medline

Navarro X, Verdú E, Wendelschafer-Crabb G, Kennedy WR (1997) Immunohistochemical study of skin reinnervation by regenerative axons. J Comp Neurol 380:164-174. CrossRef Medline

Nishiyama M, von Schimmelmann MJ, Togashi K, Findley WM, Hong K (2008) Membrane potential shifts caused by difusible guidance signals direct growth-cone turning. Nat Neurosci 11:762-771. CrossRef Medline

Obata K, Yamanaka H, Kobayashi K, Dai Y, Mizushima T, Katsura H, Fukuoka T, Tokunaga A, Noguchi K (2004) Role of mitogen-activated protein kinase activation in injured and intact primary afferent neurons for mechanical and heat hypersensitivity after spinal nerve ligation. J Neurosci 24:10211-10222. CrossRef Medline

Perlson E, Hanz S, Ben-Yaakov K, Segal-Ruder Y, Seger R, Fainzilber M (2005) Vimentin-dependent spatial translocation of an activates MAP kinase in injured nerve. Neuron 45:715-726. CrossRef Medline

Pieraut S, Laurent-Matha V, Sar C, Hubert T, Méchaly I, Hilaire C, Mersel M, Delpire E, Valmier J, Scamps F (2007) NKCC1 phosphorylation stimulates neurite growth of injured adult sensory neurons. J Neurosci 27: 6751-6759. CrossRef Medline

Pieraut S, Lucas O, Sangari S, Sar C, Boudes M, Bouffi C, Noel D, Scamps F (2011) An autocrine neuronal interleukin-6 loop mediates chloride accumulation and NKCC1 phosphorylation in axotomized sensory neurons. J Neurosci 31:13516-13526. CrossRef Medline

Price TJ, Cervero F, Gold MS, Hammond DL, Prescott SA (2009) Chloride regulation in the pain pathway. Brain Res Rev 60:149-170. CrossRef Medline

Raivich G, Makwana M (2007) The making of successful axonal regeneration: genes, molecules and signal transduction pathways. Brain Res Rev 53:287-311. CrossRef Medline

Raman M, Chen W, Cobb MH (2007) Differential regulation and properties of MAPKs. Oncogene 26:3100-3112. CrossRef Medline

Rishal I, Fainzilber M (2010) Retrograde signaling in axonal regeneration. Exp Neurol 223:5-10. CrossRef Medline

Rishal I, Fainzilber M (2014) Axo-soma communication in neuronal injury. Nat Rev Neurosci 15:32-42. CrossRef Medline

Rossi F, Gianola S, Corvetti L (2007) Regulation of intrinsic neuronal properties for axon growth and regeneration. Prog Neurobiol 81:1-28. CrossRef Medline

Sung KW, Kirby M, McDonald MP, Lovinger DM, Delpire E (2000) Abnormal $\mathrm{GABA}_{\mathrm{A}}$ receptor-mediated currents in dorsal root ganglion neurons isolated from Na-K-2Cl cotransporter null mice. J Neurosci 20:7531-7538. Medline

Torres-Espín A, Santos D, González-Pérez F, del Valle J, Navarro X (2014) Neurite-J: an Image-J plug-in for axonal growth analysis in organotypic cultures. J Neurosci Methods 236:26-39. CrossRef Medline

Waetzig V, Zhao Y, Herdegen T (2006) The bright side of JNKs-Multitalented mediators in neuronal sprouting, brain development and nerve fiber regeneration. Prog Neurobiol 80:84-97. CrossRef Medline

Waxman SG, Zamponi GW (2014) Regulating exitability of pheripheral afferents: emerging ion cannel targets. Nat Neurosci 17:153-163. CrossRef Medline

Wolf JA, Stys PK, Lusardi T, Meaney D, Smith DH (2001) Traumatic axonal injury induces calcium influx modulation by tetrodotoxin sensitive sodium channels. J Neurosci 21:1923-1930. Medline

Zrouri H, Le Goascogne C, Li WW, Pierre M, Courtin F (2004) The role of MAP kinases in rapid gene induction after lesioning the rat sciatic nerve. Eur J Neurosci 20:1811-1828. CrossRef Medline 